\title{
LES ARVICOLIDÉS (RODENTIA, MAMMALIA) DU VILLANYEN RÉCENT DE CASABLANCA I (CASTELLON, ESPAGNE)
}

\author{
par \\ JAVIER ESTEBAN AENLLE * \& NIEVES LOPEZ MARTINEZ *
}

RÉSUMÉ

L'étude de 3 espèces d'Arvicolidés de la succession de Casablanca (CSB I Castellón, Espagne) $M$. tornensis, $M$. medasensis et $M$. aff. rex permet d'établir une comparaison détaillée entre les Arvicolidés du Villanyen récent et du Biharien ancien d'Espagne, France et Europe Centrale. Une nouvelle diagnose est proposée pour $M$. tornensis; les rapports entre $M$. medasensis, $M$. pliocaenicus et $M$. ostramosensis sont discutés ; une différenciation géographique est montrée chez $M$. rex d'Espagne, France et Europe Centrale. Finalement on propose une succession biochronologique pour la période considérée, où l'on peut corréler CSB I avec les faunes de la fin du Villanyen, proches de la limite Pliocène/Pléistocène.

\section{ABSTRACT :}

The study of the three species of Arvicolids from the stratigraphical succession of Casablanca I (CSB I, Castellón, Spain) Mimomys tornensis, M. medasensis and $M$. aff. rex allows to compare in detail the Late Villanian and Early Biharian Arvicolids from Spain, France and Central Europe. A new diagnosis for $M$. tornensis is proposed : the relationships between $M$. medasensis, $M$. pliocaenicus and $M$. ostramosensis are discussed ; a geographic differentiation of $M$. rex populations from Spain, France and Central Europe is established. Finally, a biochronological succession is proposed for this period, and CSB I is correlated with the latest Villanyan faunes, near the PlioPleistocene boundary.

MOTS-CLÉS : MICROMAMMIFÈRES, ARVICOLIDÉS, MIMOMYS, VILLANYEN, LIMITE PLIO-PLÉISTOCÈNE, ESPAGNE.

KEY-WORDS : MICROMAMMALS, ARVICOLIDS, MIMOMYS, VILLANYAN, PLIO-PLEISTOCENE BOUNDARY, SPAIN.

\footnotetext{
* Departamento de Paleontologia, Facultad de C. Gzológicas, Universidad Complutense de Madrid, 28040 Madrid, Espagne.
} 

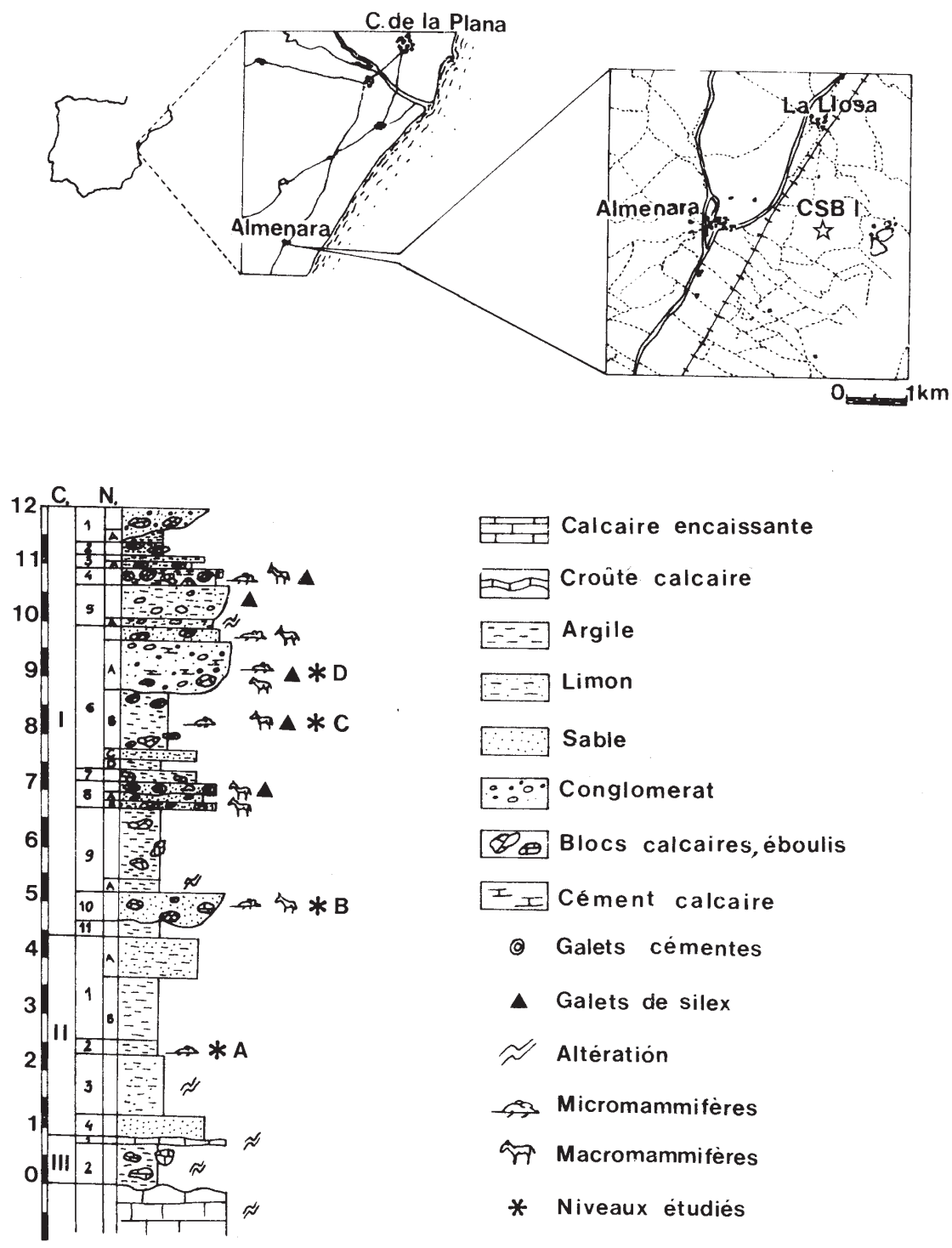

Fig. 1 - Situation géographique du gisement de CSB I et succession stratigraphique du remplissage de la fissure karstique (relevé de E. Carbonell et $\mathbf{J}$. Martinez).

Geographical situation of CSB I and stratigraphic log of the fissure (after E. Carbonell and J. Martinez). 
TABLE DES MATIÈRES

Introduction $\ldots \ldots \ldots \ldots \ldots \ldots \ldots \ldots \ldots$ p. 593

Matériel et méthodes

p. 594

Systématique

p. 596

Mymomys tornensis

p. 596

p. 601
Mymomys rex................... p. 606

Mymomys aff. rex .................. p. 606

Ulstrastructure de l'émail . .............. p. 610

Résultats et conclusions . . . . . . . . . ..... p. 610

Références bibliographiques

\section{INTRODUCTION}

\begin{tabular}{|c|c|c|c|c|}
\hline NIVEAUX & $\mathrm{CIIN}_{2}$ & Bin 10 & $\begin{array}{c}\mathrm{C} \\
\mathrm{CIN6B}\end{array}$ & $\begin{array}{c}D \\
\text { CIN6A }\end{array}$ \\
\hline $\begin{array}{l}\text { INSECTIVORA } \\
\text { Soricidae indet. } \\
\text { Crocidurinae indet.. } \\
\text { Desmana inflata RUMKE } 1985\end{array}$ & $\begin{array}{l}+ \\
+ \\
+\end{array}$ & $\begin{array}{l}+ \\
+ \\
+\end{array}$ & + & $\begin{array}{l}+ \\
+ \\
+\end{array}$ \\
\hline $\begin{array}{l}\text { CHIROPTERA } \\
\text { Myotis sp. } 1 \\
\text { Myotis sp. } 2 \\
\text { Myotis cf. myotis(BORKHAUSEN 1797) } \\
\text { Miniopterus sp. } \\
\text { Miniopterus aff. schreibersi(KUHL 1819) } \\
\text { Rhinolophus sp. } \\
\text { Rhinolophus cf. mehelyi MATSCHIE } 1901\end{array}$ & $\begin{array}{l}+ \\
+ \\
+ \\
+ \\
+\end{array}$ & $\begin{array}{l}+ \\
+ \\
+ \\
+\end{array}$ & & + \\
\hline $\begin{array}{l}\text { RODENTIA } \\
\text { Gliridae } \\
\text { Eliomys sp. } \\
\text { Muridae } \\
\text { Stephanomys progressus CORDY } 1976 \\
\text { Apodemus cf. myst acinus DANFORD \& ALSTON } 1877 \\
\text { Apodemus aff. occitanus PASQUIER } 1974 \\
\text { Castillomys crusafonti sSp. } \\
\text { Arvicolidae } \\
\text { Mimomys tornensis JANOSSY \& V. MEULEN } 1975 \\
\text { Mimomys medasensis MICHAUX 1971 } \\
\text { Mimomys aff.rex KORMOS } 1934\end{array}$ & $\begin{array}{l}+ \\
+ \\
+ \\
+ \\
+ \\
+ \\
+\end{array}$ & $\begin{array}{l}+ \\
+ \\
+ \\
+ \\
+ \\
+\end{array}$ & $\begin{array}{l}+ \\
+ \\
+\end{array}$ & $\begin{array}{l}+ \\
+ \\
+\end{array}$ \\
\hline $\begin{array}{l}\text { LAGOMORPHA } \\
\text { Prolagus sp. } \\
\text { Prolagus calpensis MAJOR } 1905\end{array}$ & + & + & & + \\
\hline
\end{tabular}

Tabl. 1 - Distribution de la faune de Micromammifères de CSB I dans les 4 niveaux étudiés. Distribution of the Micromammal fauna of CSB I in the four studied levels. 
Le gisement Villanyen de Casablanca I (CSB I) se trouve dans une carrière abandonnée, à 5 kilomètres à l'Est du village d'Almenara dans la province de Castellón (Espagne) (fig. 1). Dans cette carrière on a trouvé plusieurs remplissages de fissures karstiques, (CSB I à IV) qui contiennent des micromammifères fossiles. Gil \& Sesé (1984, 1985), Esteban (1985) et Agusti \& Galobart (1986) ont identifié et étudié des associations faunistiques de plusieurs niveaux, dont les âges se rangent depuis le Ruscinien jusqu'au Biharien.

CSB I fut découvert par les préhistoriens E. Carbonell et F. Gusi qui réalisèrent en 1983 des fouilles archéologiques. Il n'a pas encore été possible de confirmer la présence d'industrie lithique fabriquée par l'homme, associée à une faune d'âge Villanyen.

Le gisement CSB I est composé de plusieurs niveaux superposés régulièrement stratifiés, très riches en restes de micromammifères (fig. 1), surtout les niveaux A (CIIN2), B (CIN10), C (CIN6B) et D (CIN6A). La faune identifiée (Esteban 1985) jusqu'à présent comprend les espèces citées tableau 1 .

Dans ce travail nous avons approfondi l'étude systématique des Arvicolidés de CSB I, qui sont les plus importants micromammifères plio-quaternaires dans les corrélations biostratigraphiques continentales. Les 3 espèces du genre Mimomys reconnues à CSB I ont été étudiées en tenant compte des caractères comme la linea sinuosa, l'ultrastructure de l'émail, la morphologie et la taille des molaires. La grande abondance du matériel a permis de décrire la forte variabilité des populations. Enfin, on discute les hypothèses phylogénétiques. On aboutit finalement à une proposition d'arrangement chronologique des gisements européens les plus importants du Pliocène supérieurPléistocène inférieur, et à une corrélation de CSB I avec eux.

\section{MATÉRIEL ET MÉTHODES}

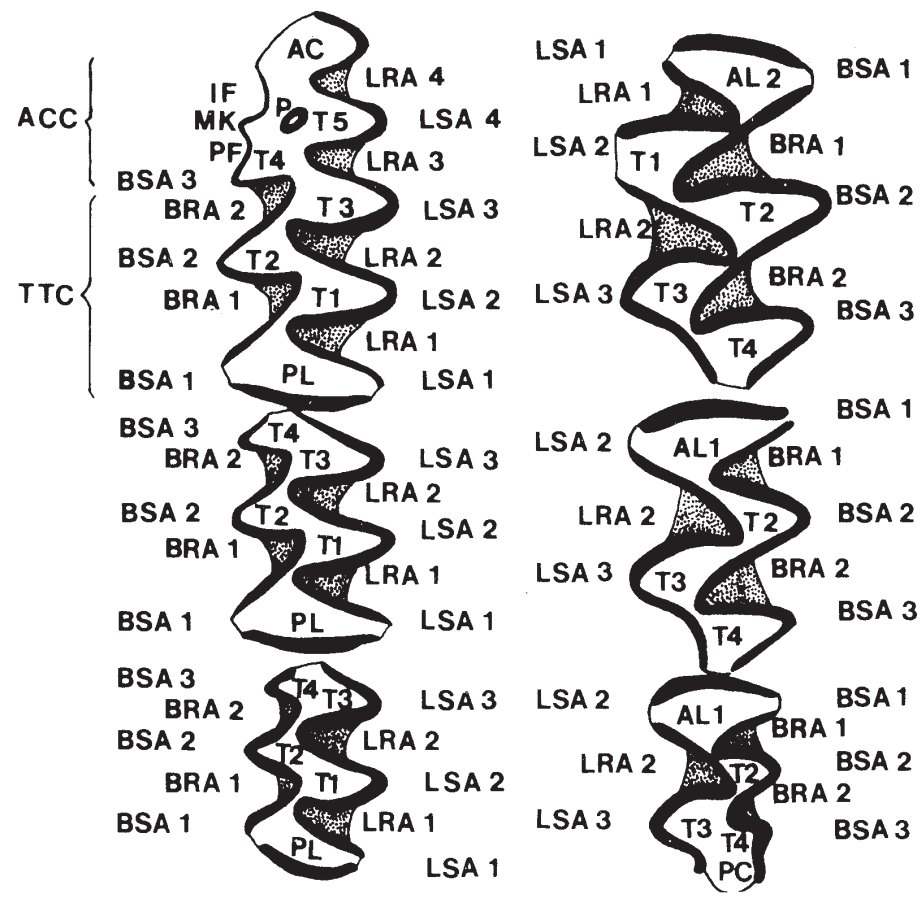

Fig. 2 - Terminologie dentaire des Arvicolidés. A gauche molaires inférieures, à droite molaires supérieures. AC, cuspide antérieure ; $\mathrm{ACC}$, complexe antéroconide ; $\mathrm{AL}$, lobe antérieur ; BRA, angle rentrant buccal ; BSA, angle saillant buccal ; IF, inselfalte ; LRA, angle rentrant lingual ; LSA, angle saillant lingual ; MK, mimomyskante ; $\mathrm{P}$, puiselet ; $\mathrm{PC}$, cuspide postérieure ; $\mathrm{PF}$, prismenfalte ; PL, lobe postérieur ; $T$, triangles d'émail ; TTC, complexe trigonidetalonide. (d'après Michaux 1971 et V. Meulen 1973).

Dental terminology of Arvicolids. Left, lower molar ; right, upper molar. AC, anterior cap ; $\mathrm{ACC}$, anteroconid complex; $\mathrm{AL}$, anterior lobe ; BRA, buccal re-entrant angle ; BSA, buccal salient angle ; IF, inselfalte ; LRA, lingual re-entrant angle; LSA, lingual salient angle; MK, mimomyskante; $P$, islet ; OC, posterior cap ; $\mathrm{PF}$, prismenfalte ; $\mathrm{PK}$, posterior lobe ; TTC, trigonid-talonid complex (after Michaux 1971 and V. Meulen 1973). 

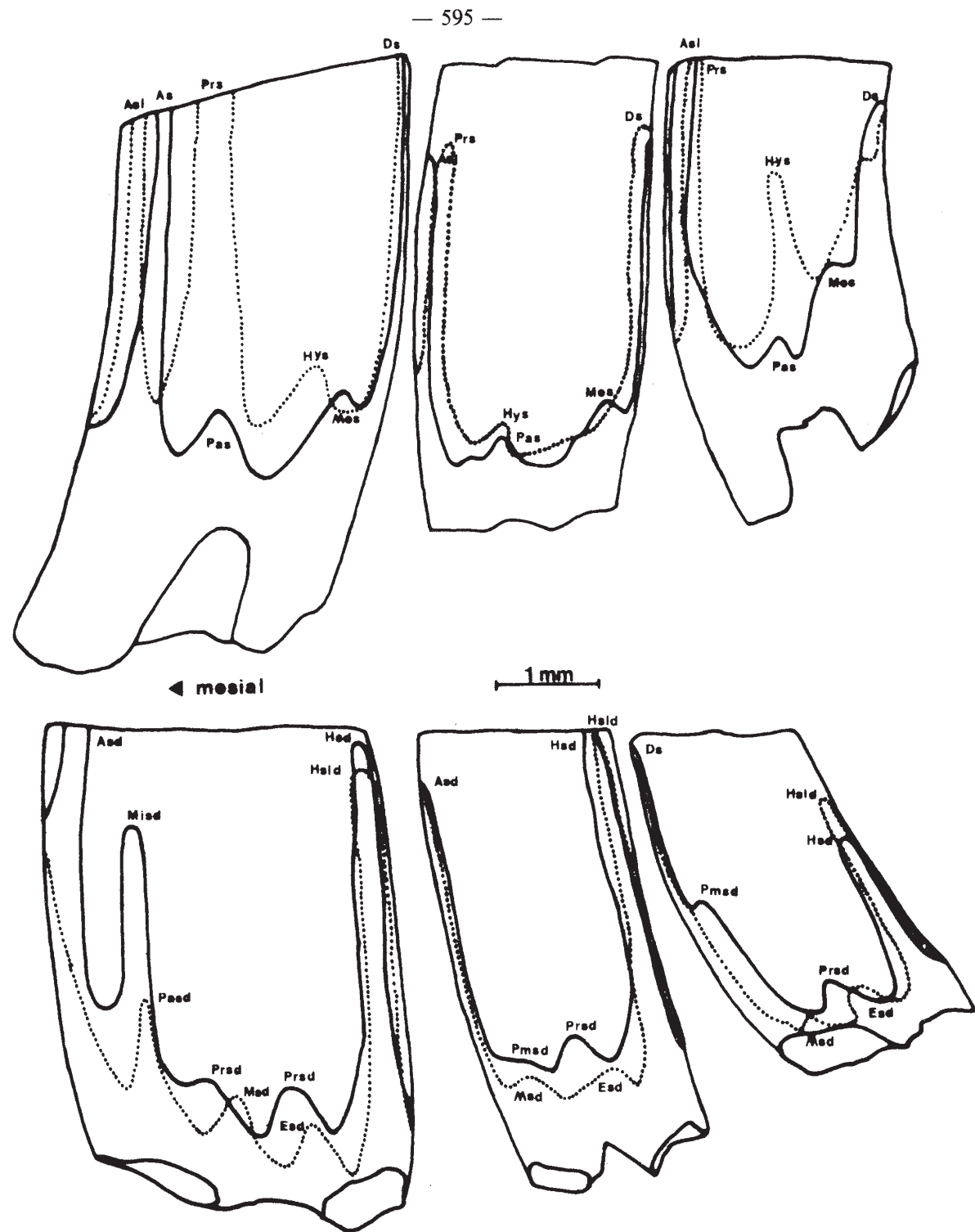

Fig. 3 - Terminologie de la linea sinuosa d'après Rabeder 1981, sur $M$. medasensis. En bas, molaires inférieures ; en haut, molaires supérieures. En trait continu, linea sinuosa de la face buccale ; en pointillé, celle de la face linguale. As, antérosinus ; Ads, antérosinuide ; Als, antérosinule ; Ds, distosinus . Esd, entosinuide ; Hsd, hyposinuide ; Hsld, hyposinulide ; Hys, hyposinus ; Mes, métasinus ; Misd, mimosinuide ; Msd, metasinuide ; Pas, parasinus ; Pasd, parasinuide ; Pmsd, prismosinuide . Prs, protosinus ; Prsd, protosinuide.

Terminology of the linea sinuosa according to Rabeder 1981, with $M$. medasensis as an exemple. Below, lower molars. Above, upper molars. Full line, the linea sinuosa of the buccal face ; broken line, that of the lingual face. As, anterosinus ; Ads, anterosinuid ; Asl, anterosinule ; Ds, distosinus ; Esd, entosinuid ; Hsd, hyposinuid ; Hsld, hyposinulid ; Hys, hyposinus ; Mes, metasinus ; Misd, mimosinuid ; Msd, metasinuid ; Pas, parasinus ; Pasd, parasinuid ; Pmsd, prismosinuid ; Prs, protosinus ; Prsd, protosinuid. 
Le sédiment extrait du gisement (quelques 60 kilogrammes au total) a été soumis au traitement de lavage-tamisage. Pour les blocs très cimentés on a suivi la technique d'attaque-acide. Parmi les restes de micromammifères, on a obtenu 533 dents appartenant au genre Mimomys, distribués comme l'on voit dans le tableau 2.

Sur toutes les molaires on a pris deux mesures à la surface occlusale : la longueur maximale et la largeur. La hauteur a été mesurée sur quelques individus. Les deux dernières variables ont été mesurées sur le lobe postérieur chez les molaires inférieures et sur l'antérieur, chez les molaires supérieures. Les mesures effectuées avec un microscope WILD M8 doté d'un réticule micrométrique sont exprimées en mm. La terminologie utilisée pour la description de la surface occlusale des molaires a été prise et modifiée de Michaux 1971 et de Van der Meulen 1973 (fig. 2); pour la linea sinuosa la terminologie suivie est celle de
Rabeder 1981 (fìg. 3). Dans l'étude de l'ultrastructure de l'émail nous avons sélectionné la technique développée par Rabeder \& Satler 1984, qui demande de faire une lame mince de 0,08 à $0,01 \mathrm{~mm}$ d'épaisseur.

\begin{tabular}{|l|c|c|c|c|}
\hline ESPECES NIVEAU & A & B & D & TOTAL \\
\hline M. tornensis & & & & \\
\hline M. medasensis & 85 & - & - & 85 \\
M. aff. rex & 130 & 197 & 27 & 354 \\
TOTAL & 10 & 79 & 5 & 94 \\
\hline
\end{tabular}

Tabl. 2 - Distribution des espèces d'Arvicolidés de CSB I dans les 3 niveaux où ils ont été enregistrés.

Distribution of the Arvicolids species from CSB I in the three levels where they have been recorded.

\section{SYSTÉMATIQUE}

Ordre RODENTIA BODWICH, 1821

Famille Arvicolidae GRAY, 1821

Genre Mimomys FORSYTH MAJOR, 1902

Mimomys tornensis JANOSSY \& VAN Der MaUlen, 1975

texte-fig. 4 ; pl. 1 , fig. 1 à 18

1975 - Mimomys tornensis n. sp. JANOSSY \& VAN Der MEulen, p. 385-386, fig. 4-7.

1981 - Mimomys tornensis JANOSSY \& VAN DER MEULEN, 1975. Rabeder, p. 172-175, fig. 108.

1983 - Mimomys (Cseria) newtoni tornensis JANOSSY \& VAN DeR MEulen, 1975. Fejfar \& Horacek, p. 143-144, taf. 9 et 10 .

\section{LOCALITÉ TYPE :}

Osztramos 3 (Hongrie).

\section{DiAGNOSE ORIGINELLE :}

«A phylogenetically advanced, medium-sized Mimomys species, characterized by molars having abundant crown cementum in the deep reentrant angles, and by the anteroconid complex lacking Mimomys ridge or islet and often bearing a fourth buccal salient angle and occasionally and incipient fourth lingual salient angle » (Janossy \& Van der Meulen, 1975, p. 385)

\section{DIAGNOSE ÉMENDÉE :}

Mimomys de taille moyenne $\left(\mathrm{LM}_{1}=2.81\right)$, avec des molaires très hypsodontes et du cément très abondant ; $\mathrm{M}_{1}$ avec mimomyskante présent dans des proportions variables et en position avancée en face du LRA 4 ; l'inselfalte, plus faible que le prismenfalte, disparaît très rapidement avec l'usure ; mimosinuide variablement développé dans la linea sinuosa. Puiselet absent. $\mathrm{M}^{3}$ avec $\mathrm{AL1}, \mathrm{T}_{2}$ et $\mathrm{T}_{3}$ séparés nettement par de profonds angles rentrants.

\section{DISTRIBUTION :}

Osztramos 3 (Hongrie) ; Deutsch-Altenburg (Allemagne) ; Ctineves, Koliňany 1, 2 et 3, Plešivec et V̌̌eláre $3,3 \mathrm{~B}$ et $3 \mathrm{~B} / 1$ (Tchécoslovaquie).

\section{MATÉRIEL DE CSB I :}

Niveau A : 2 mandibules inférieures complètes, 3 mand. inférieures dépourvues de $\mathrm{M}_{3}, 21 \mathrm{M}_{1}, 18 \mathrm{M}_{2}$ et $10 \mathrm{M}_{3} ; 11 \mathrm{M}^{1}, 8 \mathrm{M}^{2}$ et $7 \mathrm{M}^{3}$. 
MENSURATIONS :

\begin{tabular}{|c|c|c|c|c|c|c|c|c|}
\hline & \multicolumn{5}{|c|}{$\mathrm{L}$} & \multicolumn{3}{|c|}{1} \\
\hline & & Min. & Moy. & Max. & $\mathrm{N}$ & Min. & Moy. & Max. \\
\hline \multirow{6}{*}{ 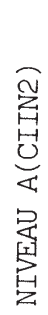 } & $M_{1}$ & 2.45 & 2.81 & 3.08 & $25 / 26$ & 0.96 & 1.15 & 1.27 \\
\hline & $M_{2}$ & 1.58 & 1.82 & 2.09 & $22 / 23$ & 0.79 & 1.00 & 1.35 \\
\hline & $\mathrm{M}_{3}$ & 1.46 & 1.55 & 1.62 & $10 / 9$ & 0.78 & 0.81 & 0.86 \\
\hline & $\mathrm{M}^{1}$ & 2.33 & 2.55 & 2.64 & 11 & 1.08 & 1.25 & 1.34 \\
\hline & $\mathrm{M}^{2}$ & 1.83 & 1.98 & 2.07 & 8 & 0.83 & 1.11 & 1.21 \\
\hline & $M^{3}$ & 1.54 & 1.70 & 1.78 & 7 & 0.88 & 0.98 & 1.07 \\
\hline
\end{tabular}

Tabl. 3 - Mesures de la longueur (L) et de la largeur (l) des dents de M. tornensis du CSB I, niveau A.

Measurements of the length (L) and width (I) of the M. tornensis teeth from CSB I, level A.

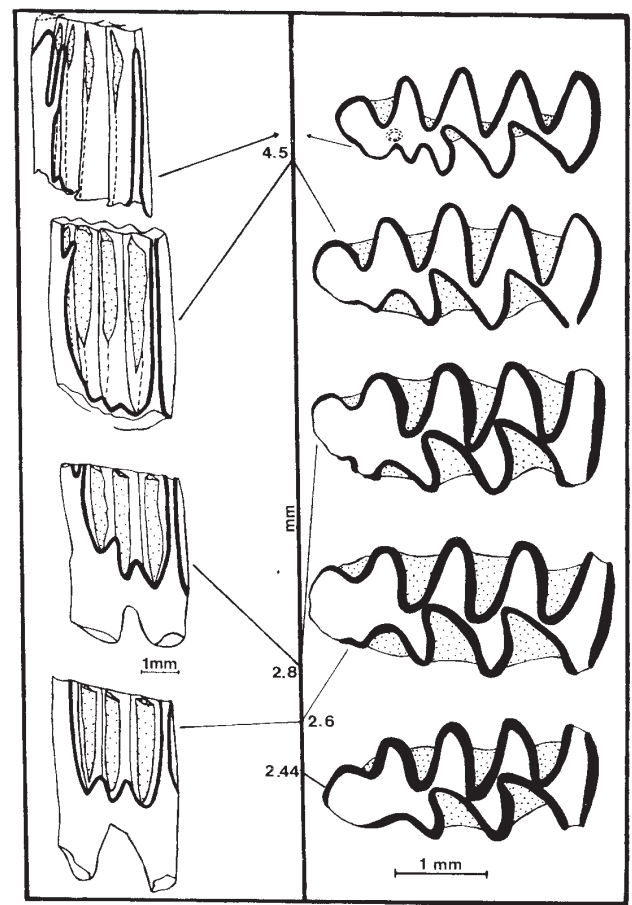

†ig. 4 - Séquence d'usure de la $\mathbf{M}_{1}$ chez $M$. tornensis de CSB $\mathbf{I}$. Wear sequence of $M$. tornensis de CSB I.

\section{DESCRIPTION :}

M1. Elles sont constituées par un complexe antéroconide (ACC), trois triangles d'émail isolés et un lobe postérieur. Dans le complexe antéroconide, le prismenfalte est moins profond que le LRA 4 ; l'inselfalte est moins développé que le prismenfalte et disparaît couramment tôt, à une hauteur supérieure à celle de la moitié de la couronne. Il y a des exemplaires avec un inselfalte relativement bien développé et souvent rempli de cément, ils correspondent à des individus assez jeunes. D'autres $\mathrm{M}_{1}$ provenant d'individus moins jeunes, montrent un très faible sillon ou une surface plate au-devant du mimomyskante, dans la même position que l'inselfalte. Ce sillon a été nommé microtusfalte par Rabeder (1981, p. 165) et sa relation avec le mimomyskante peut être observée dans la séquence d'usure de la $M_{1}$ de notre population (fig. 4) et dans la figure 5. Dans tous les exemplaires, le prismenfalte est le plus développé des sillons labiaux de l'ACC ; on n'observa pas de puiselet.

Le mimomyskante, à cause du grand développement du prismenfalte, a une position relativement avancée, presqu'en face du LRA 4 . Il est peu marqué quand l'inselfalte disparaît, et peut être complètement absent sur des exemplaires très usés. Sur toutes les molaires le cément est très épais.

La linea sinuosa présente trois morphotypes selon le développement du mimosinuide (fig. 5) : a) réduit, b) petit, et c) bien développé. Les interruptions d'émail peuvent être observées dans les dents très jeunes, dans 
la partie antéro-externe de la cuspide antérieure et aux côtes du lobe postérieur.

$\mathbf{M}_{2}$. Aux dents adultes, les triangles 1 et 2 confluent largement. Sur la linea sinuosa (fig. 6), l'antérosinuide, l'hyposinuide et l'hyposinulide sont très développés.
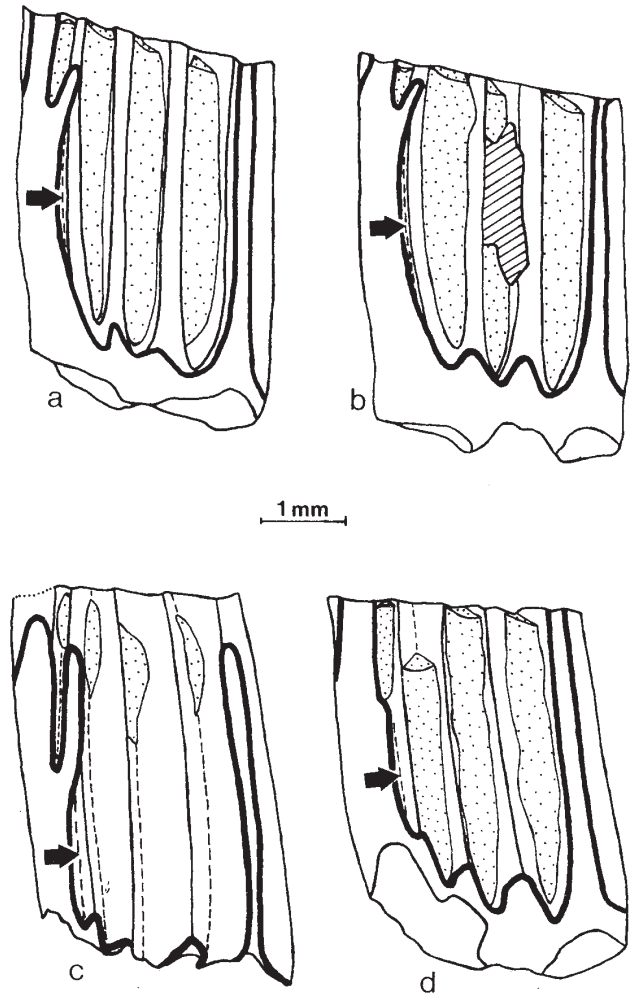

Fig. 5 - Différents degrés de dévaloppement du mimosinuide chez $M_{\circ}$ tornensis de CSB

a) et b), mimosinuide petit c) bien développé et d) réduit. La flèche montre le sillon nommé microtusfalte par Rabeder (1981), qui continue l'inselfalte en dessous du mimosinuide, au-devant du mimomyskante.

Degrees of development of the mimosinuide in $M$. tor nensis from CSB I.

a) and b) small. c) well-developed and d) reduced. Arrow shows the furrow named microtusfalte by Rabeder (1981) which follows the inselfalte below the mimosinuide, before the mimomyskante.
M3. Les triangles d'émail sont largement confluents. T1 et T2 sont presque opposés ainsi que T3 et T4. L'émail est interrompu sur la face antérieure de la dent et sur les côtés interne et externe du lobe postérieur.

M1. Les triangles d'émail sont isolés. On observe des interruptions de l'émail au lobe antérieur, au T1 et à la pointe postérieure du T4. Sur un total de huit dents, cinq présentent deux racines et les autres en ont trois.

$\mathbf{M}^{2}$. Aux dents adultes, les triangles se trouvent isolés ou légèrement confluents. Sur la linea sinuosa (fig. 6) se développent l'antérosinule et le distosinus ; par conséquent les interruptions de l'émail s'observent sur des dents très jeunes à la pointe postérieure du T4 et des deux côtés du lobe antérieur.

$\mathbf{M}^{3}$. T2 et T3 sont fermés sur les dents très usées qui ont perdu le puiselet et communiquent très légèrement dans les autres cas ; cela est dû à l'angle rentrant labial 2 (LRA 2) qui, très profond, est en contact avec l'émail du côté buccal. T3 et T4 communiquent fortement. T5 est un triangle à peine développé, très ouvert. Les angles rentrants externes sont orientés transversalement ; BRA 2 est fortement recourbé vers la partie postérieure chez les dents adultes dépourvues du puiselet; BRA 3 est peu prononcé et très ouvert. Le puiselet sur la cuspide postérieure est présent sur les exemplaires juvéniles, et disparaît sur les adultes qui ont de larges interruptions de l'émail (pl. 1, fig. 15 à 18). LRA 3, peu profond sur les exemplaires jeunes, disparaît avec l'usure.

L'émail est très différencié sur les dents provenant d'individus adultes. Sur la linea sinuosa se développent l'antérosinule, le protosinus et le distosinus.

\section{DisCUSSION :}

Le plus petit Mimomys de CSB I se rapproche d'une espèce très évoluée, d'après sa grande hypsodontie, le grand développement des sinus à la linea sinuosa, l'abondance du cément et l'absence de puiselet aux $M_{1}$. Ces caractères évolués dans une population de la même taille que celle de CSB I se trouvent chez $M$. tornensis JANOSSY \& V. MEULEN, 1975 du Villanyen récent d'Osztramos 3 (Hongrie) 1

Dans la diagnose originelle de $M$. tornensis les auteurs indiquent l'absence de mimomyskante mais la présence occasionnelle d'un 4ème angle saillant buccal (BSA 4) (Janossy \& Van der Meulen 1975, pl. 1, fig. 4a). A notre avis, cette crête est un vrai mimomyskante en position avancée. Le problème est posé quand 

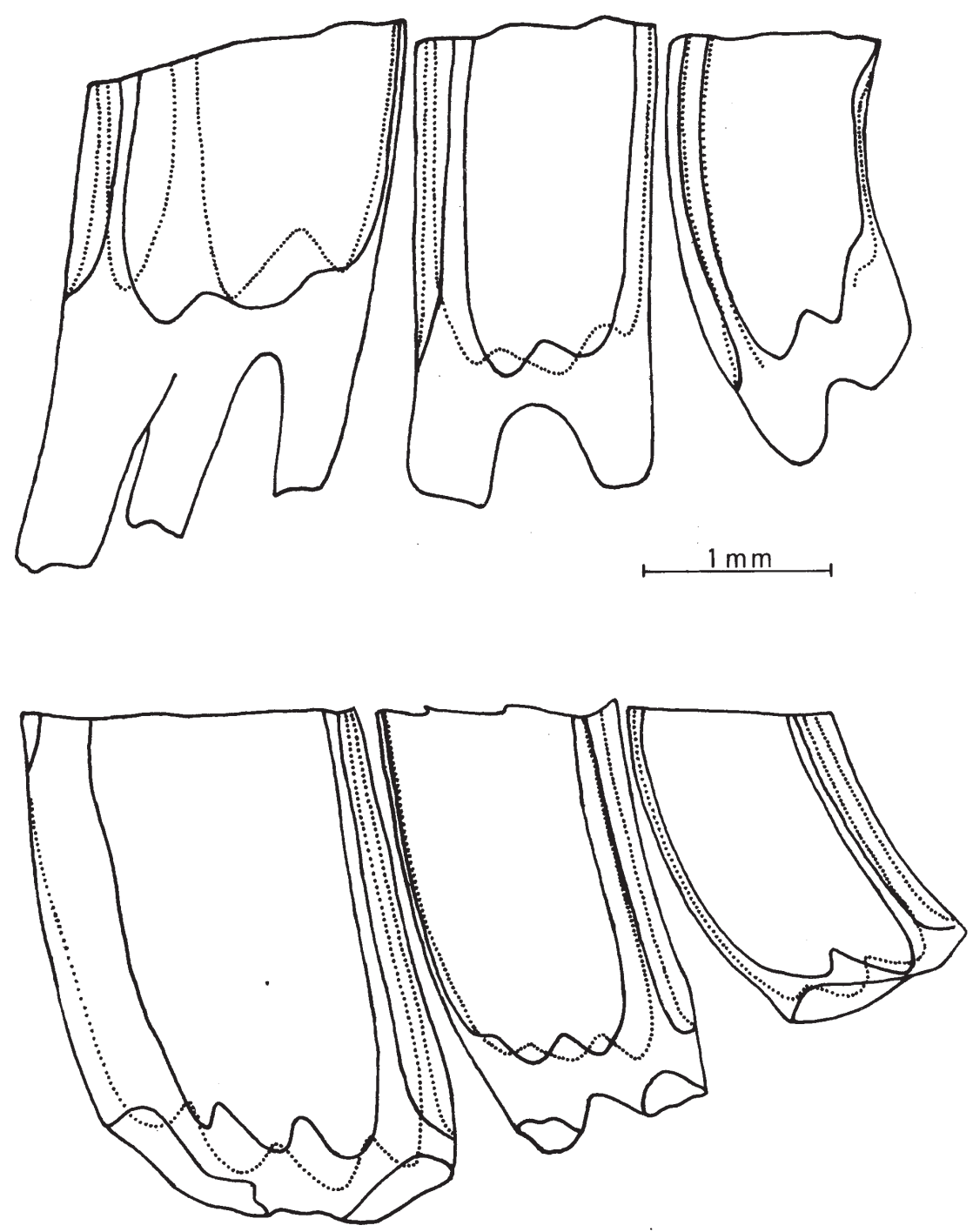

Fig. 6 - Linea sinuosa chez M. tornensis de CSB I. En bas les molaires inférieures, en haut, les supérieures. Linea sinuosa of M. tornensis from CSB I. Below, lower molars ; above, upper molars. 
Van der Meulen 1973 (p. 40-41, pl. 5) considère que chez $M$. blanci le mimomyskante se trouverait seul dans les stades très juvéniles et disparaîtrait rapidement. Cependant nos observations indiquent qu'un repli juvénile éphémère, considéré par Meulen comme le mimomyskante, peut apparaître sur l'angle BSA 3 dans diverses espèces ( $n^{\circ} 1$ dans la fig. 7) étant cependant bien différencié du mimomyskante qui est antérieur ( $\mathrm{n}^{\circ} 2$ dans la fig. 7, considéré par Van der Meulen comme un BSA 4). En vue latérale (fig. 7) on peut vérifier que le repli $n^{\circ} 1$ disparaît très rapidement avec l'usure et qu'il y a une continuité totale entre le repli $\mathrm{n}^{\circ} 2$ et le mimomyskante des stades adultes. Cela implique une modification de la diagnose originale de $M$. tornensis, mais ne jette pas de doute sur la ressemblance des populations d'Osztramos 3 et CSB I.
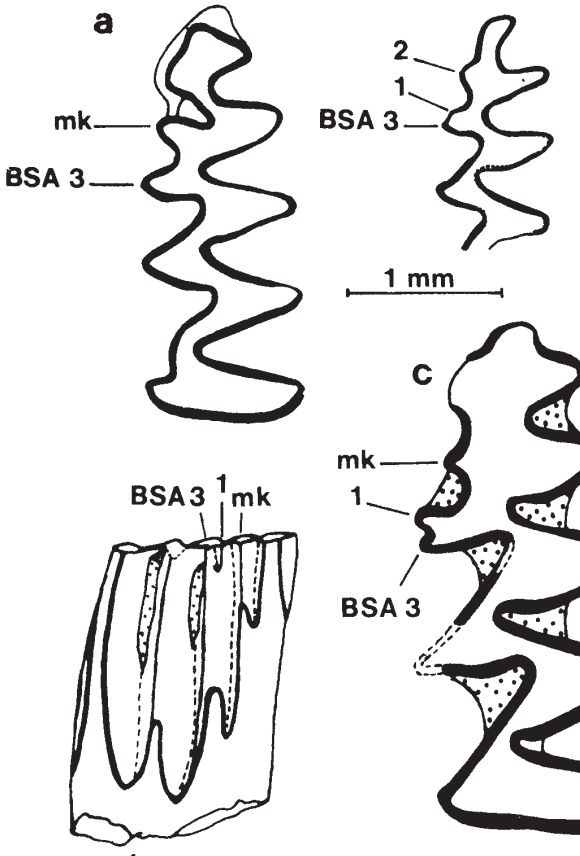

b

$1 \mathrm{~mm}$

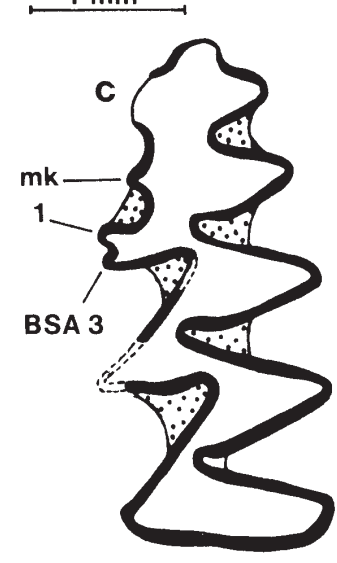

$1 \mathrm{~mm}$

Fig. 7 - Interprétation des stades juvéniles de la $\mathbf{M}_{\mathbf{1}}$ de Mimomys ; a) M. pusillus selon V. Meulen, 1973, fig. $14 \mathrm{a}$, p. 40 ; b) M. blanci V. Meulen 1973, pl. 5, fig. 2 ; c) $M$. medasensis de CSB I, vue occlusale et latérale. Mk, mimomyskante ; BSA 3, 3ème angle saillant buccal. Le repli $n^{\circ} 1$ chez $M$. blanci considéré par V. Meulen comme le mimomyskante est aussi présent dans $M$. medasensis juvénile, et on peut le voir disparaître en vue latérale derrière le mimomyskante. Le repli homologue du mimomyskante n'est donc pas le $n^{\circ} 1$, mais le $n^{\circ} 2$ qui était nommé BSA 4 par Janossy \& V. Meulen (1975) dans la diagnose de $M$. tornensis.
Rabeder (1981) a rapporté quelques exemplaires en provenance de Deutsch-Altenburg à $M$. tornensis. D'après son interprétation l'inselfalte est remplacé par un autre sillon nommé microtusfalte situé dans la même position (voir description de la population de CSB I et fig. 5). Selon Rabeder (1981, p. 174), le mimomyskante existerait jusqu'au remplacement de l'inselfalte par le microtusfalte et, ceci réalisé, il s'appellerait microtuskante. Cependant, comme il y a continuité de la crête pendant le remplacement des sillons, il ne semble pas nécessaire de changer de nom.

Fejfar \& Horacek (1983) décrivent sous le nom de $M$. newtoni tornensis, des populations de Ctiněves, Koliňany 1,2,3, Plešivec, Včelare 3,3B, 3B/1. Ils notent la présence de mimomyskante dans des pourcentages qui varient entre 11 et 14,5\%. D'après leurs figures, on peut supposer que l'absence d'inselfalte a été comptabilisée avec les absences de mimomiskante. Si l'on considère leurs figures 14 à 18 (planche 9), et 10 à 13 (planche 10) comme des formes avec mimomiskante (même si elles n'ont pas d'inselfalte) la fréquence augmenterait. De même dans la population type nous pouvons reconnaître des spécimens avec mimomyskante sans inselfalte (Janossy \& V. Meulen 1975, pl. 1, fig. 6 et 7).

Par conséquent, la présence de mimomyskante avec une certaine fréquence étant suffisamment documentée, il devenait nécessaire de changer la diagnose. Aussi, dans la diagnose originelle on cite la présence occasionnelle d'un LSA 4, ce qui doit être une erreur, car le LSA 4 est toujours présent chez tous les Mimomys.

Chez le $M$. tornensis de CSB I, l'inselfalte manque sur $44 \%$ des $M_{1}$ mais on observe un mimomyskante sur $76 \%$ d'entre elles. Les $M_{1}$ qui ne le présentent pas sont toujours des exemplaires très vieux ou moyennement usés où il est possible que le mimomyskante ait existé dans des stades plus récents d'usure ; si la fréquence de ces caractères varie selon l'âge des individus il n'est pas possible de comparer des pourcentages sans connaître la composition en classes d'âges des populations de $M$. tornensis. La présence de trois

Interpretation of the Mimomys young $\mathbf{M}_{1}$. a) $M$. pusillus from V Meulen 1973, fig. 14a, p. 40 ; b) M. blanci V. Meulen, 1973, pl. 5, fig. 2 ; c) $M$. medasensis from CSB I, occlusal and lateral view. MK, mimomyskante, BSA 3, third buccal salient angle. The ridge number 1 of $M$. blanci, considered the mimomyskante. Consequently, the mimomyskante is not the ridge $n^{\circ} 1$, but the $n^{\circ} 2$ named BSA 4 by Janossy \& V. Meulen 1975 in the diagnosis of $M$. tornensis. 

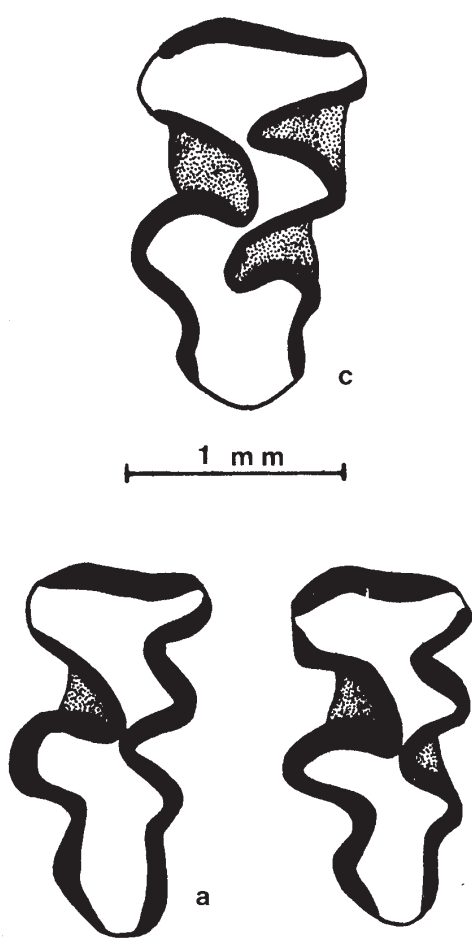

Fig. 8 - Comparaison des $\mathbf{M}^{\mathbf{3}}$ de $\boldsymbol{M}$. pusillus de Deutch Altenburg (figs. a et b, d'après Rabeder 1981) et $M$. tornensis de CSB I (fig. c). Les degrés d'usure sont semblables dans les trois cas.

Comparison of the $\mathrm{M}^{3}$ of $M$. pusillus from Deutch Altenburg (figs. a and b, according to Rabeder, 1981) and $M$. tornensis from CSB I (fig. c). Similar wear state in the three cases.

racines s'observe chez $27 \%$ de $M^{1}$ de notre population. Ce trait est primitif par rapport à la présence constante de deux racines. Dans la localité type d'Osztramos 3, la présence de deux racines est indiquée mais le nombre d'exemplaires n'étant pas fourni, il est difficile de conclure sur l'état de ce caractère.

D'autres espèces de faible taille montrent des ressemblances avec $M$. tornensis. $M$. newtoni MAJOR, 1902 a été proposé comme son ancêtre possible (Janossy \& Van der Meulen 1975) ; d'autres auteurs incluent ces deux espèces dans une seule (Fejfar \& Horacek 1983), ce qui nous semble encore prématuré, parce que $M$. newtoni décrit à partir de peu d'exemplaires par Hinton (1926), n'est pas bien caractérisé et on ne connaît pas la variabilité de la population-type. On rapporte à $M$. newtoni une population bien représentée de Tegelen (Freudenthal et alii 1976). A Kadzielnia, Kowalski (1958) signale une population décrite comme $M$. newtoni qui pourrait appartenir à $M$. tornensis. $M$. newtoni a été cité à Chagny (Chaline $\&$ Michaux 1974) et à Gundersheim (Heller 1936). A Okrà 1, Fejfar \& Horacek (1983) signalent le plus récent $M$. cf. newtoni mais ils considèrent $M$. newtoni et $M$. tornensis comme une seule espèce. Tous les auteurs figurent des $M_{1}$ sans mimomyskante et sans inselfalte, sauf Heller qui figure une dent très jeune avec mimomyskante et inselfalte attribuée à $M$. newtoni.

M. pusillus Menely, 1914, ressemble à M. tornensis par la morphologie des $\mathrm{M}_{1}$ et la taille, tout en montrant cependant des différences claires. $M$. tornensis présente une taille un peu plus grande (fig. 8), le cément est plus développé ; le prismenfalte, rempli de cément, est plus large que dans $M$. pusillus (Rabeder 1981) et se fait encore plus profond avec l'usure. $\mathrm{T}_{1}$ et $\mathrm{T}_{2}$ confluent chez $M$. pusillus. Des différences encore plus accusées s'observent chez les $\mathrm{M}^{3}$ (fig. 8) : $M$. pusillus présente des triangles plus confluents (AL1 et T2 confluent largement) ; BRA 1 et BRA 2 sont moins profonds et s'orientent transversalement à l'axe antéro-postérieur de la dent; LRA 3 est plus développée que chez $M$. tornensis. Dans celui-ci, BRA 1 et BRA 2 sont plus profonds sur les $\mathrm{M}^{3}$ adultes qui ont perdu le puiselet, et BRA 2 est fortement incliné vers la partie postérieure.

Mimomys medasensis MICHAUX, 1971

texte-fig. 9 ; pl. 2 , fig. 1 à 21

1971 - Mimomys medasensis n.sp. : Michaux, p. 191-197.

1978 - Mimomys medasensis Michaux : Mein, Moissenet \& Truc, p. 112-122.

1982 - Mymomys cf. cappettai : Alberdi et alii, p. 263.

\section{LOCALITÉ TYPE :}

Iles Medas, île Meda Grande (Gerona, Espagne).

\section{DIAGNOSE ORIGINELLE :}

«Mimomys d'assez grande taille. Lobe antérieur des $\mathrm{M}_{1}$ comprimé transversalement. $\mathrm{M}^{1}$ a deux 
racines, $\mathrm{M}_{1}$ avec puiselet fugace. Hypsodontie forte, présence de cément, échancrures de l'émail importantes » (Michaux 1971, p. 192).

\section{DISTRIBUTION :}

Iles Medas, Valdeganga I et II, El Rincón 1 (Espagne).
MATÉRIEL DE CSB I :

Niveau A : 2 mandibules inférieures complètes et 2 incomplètes, $22 \mathrm{M}_{1}, 24 \mathrm{M}_{2}$ et $12 \mathrm{M}_{3} ; 22 \mathrm{M}^{1}, 23 \mathrm{M}^{2}$ et $19 \mathrm{M}^{3}$.

Niveau $\mathrm{B}: 37 \mathrm{M}_{1}, 41 \mathrm{M}_{2}$ et $36 \mathrm{M}_{3} ; 32 \mathrm{M}^{1}, 29 \mathrm{M}^{2}$ et $22 \mathrm{M}^{3}$.

Niveau D : $6 \mathrm{M}_{1}, 6 \mathrm{M}_{2}$ et $4 \mathrm{M}_{3} ; 5 \mathrm{M}^{1}, 3 \mathrm{M}^{2}$ et 3 $\mathrm{M}^{3}$.

\section{MEnSuRATions :}

\begin{tabular}{|c|c|c|c|c|c|c|c|c|}
\hline & & & L & & & & 1 & \\
\hline & & Min. & Moy. & Max. & $\mathrm{N}$ & Min. & Moy. & Max. \\
\hline \multirow{6}{*}{ 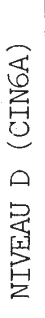 } & $\mathrm{M}_{1}$ & 2.84 & 3.36 & 3,75 & 6 & 1.22 & 1.44 & 1.58 \\
\hline & $\mathrm{M}_{2}$ & 1.93 & 2.11 & 2.25 & 6 & 1.00 & 1.26 & 1.50 \\
\hline & $\mathrm{M}_{3}$ & 1.54 & 1.70 & 1.80 & 4 & 0.68 & 0.89 & 1.03 \\
\hline & $\mathrm{M}^{\top}$ & 2.84 & 2.88 & 2.92 & 5 & 1.30 & 1.37 & 1.44 \\
\hline & $M^{2}$ & 2.21 & 2.26 & 2.29 & 3 & 1.30 & 1.34 & 1.38 \\
\hline & $\mathrm{M}^{3}$ & 2.00 & 2.12 & 2.35 & 3 & 1.13 & 1.17 & 1.23 \\
\hline \multirow{6}{*}{ 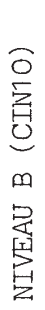 } & $M_{1}$ & 2.80 & 3.42 & 3.95 & 37 & 1.10 & 1.43 & 1.75 \\
\hline & $\mathrm{M}_{2}$ & 1.87 & 2.19 & 2.41 & $40 / 41$ & 0.86 & 1.33 & 1.58 \\
\hline & $\mathrm{M}_{3}$ & 1.42 & 1.84 & 2.27 & $36 / 34$ & 0.61 & 0.96 & 1.30 \\
\hline & $\mathrm{M}^{1}$ & 2.64 & 3.02 & 3.55 & 32 & 1.14 & 1.38 & 1.73 \\
\hline & $\mathrm{M}^{2}$ & 2.19 & 2.32 & 2.49 & 29 & 1.02 & 1.33 & 1.62 \\
\hline & $\mathrm{M}^{3}$ & 1.55 & 1.94 & 2.38 & 22 & 0.74 & 1.16 & 1.34 \\
\hline \multirow{6}{*}{ 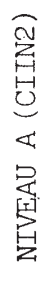 } & M1 & 2.84 & 3.23 & 3.47 & $21 / 22$ & 1.14 & 1.36 & 1.54 \\
\hline & $\mathrm{M}_{2}$ & 1.97 & 2.15 & 2.29 & $24 / 23$ & 1.02 & 1.27 & 1.46 \\
\hline & $\mathrm{M}_{3}$ & 1.40 & 1.74 & 1.97 & 20 & 0.63 & 0.89 & 1.10 \\
\hline & $\mathrm{M} 1$ & 2.39 & 2.85 & 3.12 & 22 & 1.00 & 1.25 & 1.54 \\
\hline & $M^{2}$ & 2.09 & 2.27 & 2.37 & 23 & 1.18 & 1.29 & 1.50 \\
\hline & $\mathrm{M}^{3}$ & 1.56 & 1.93 & 2.17 & 19 & 0.94 & 1.09 & 1.23 \\
\hline
\end{tabular}

Tabl. 4 - Mesures de la longueur (L) et la largeur (l) des dents de $M$. medasensis de CSB I, niveau A,B et D.

Measurements of the length (L) and width (l) of the M. medasensis teeth from CSB I, levels A, B and D. 


\section{DESCRIPTION :}

On observe deux racines à toutes les molaires; le cément est assez développé. Les dents adultes montrent un émail différencié épaissi sur le bord antérieur des triangles aux dents supérieures, et sur le bord postérieur aux dents inférieures. Elles sont très hypsodontes.

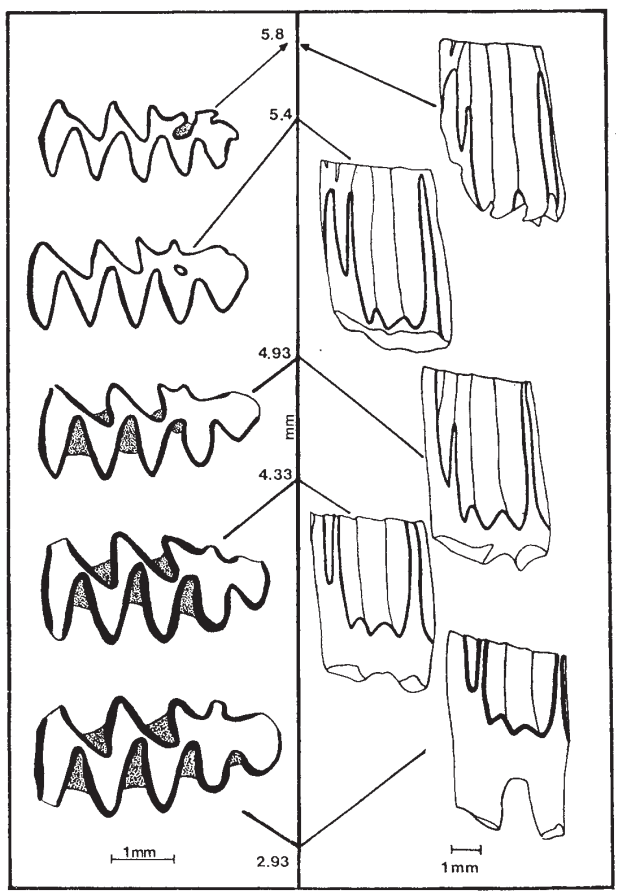

Fig. 9 - Séquence d'usure de la $\mathbf{M}_{1}$ chez $M$. medasensis de CSB

Wear sequence of $M$. medasensis $M_{1}$ from CSB $I$.

M1. Sur le complexe antéroconide, l'inselfalte et le prismenfalte sont très ouverts et peu profonds. Le mimomyskante peut être observé sur toute la hauteur de la couronne. Le puiselet disparaît très vite avec l'usure, on n'en voit presque pas sur les dents adultes à émail différencié (fig. 9).

Le LRA 4 est très profond mais sur les dents usées il s'atténue. Les triangles d'émail du côté lingual sont plus développés que ceux du côté buccal, ce qui donne un dessin très nettement dissymétrique. T1 et T2 confluent sur les dents jeunes, et s'isolent sur les dents très usées. Les synclinaux sont orientés transversalement, leurs pointes étant légèrement recourbées vers l'avant.

Sur la linea sinuosa (fig. 3), l'antérosinuide descend en oblique sur le bord antérieur ; le mimosinuide est très développé, atteignant la moitié de la hauteur de la couronne, et laissant une interruption de l'émail sur le mimomyskante ; au lobe postérieur, l'hyposinuide et l'hyposinulide atteignent la surface occlusale, même sur les dents jeunes.

M2. T1 et T2 communiquent, T2 et T3 sont pratiquement isolés. L'antérosinuide, l'hypsosinuide et l'hyposinulide sont très développés (fig. 3).

M3. T1-T2 et T3-T4 communiquent sur les dents usées jusqu'à l'opposer totalement. Les LRA sont plus profonds que les BRA, les premiers s'orientent transversalement à l'axe antéropostérieur de la dent, tandis que les seconds sont légèrement inclinés vers la partie antérieure.

Le dessin de la linea sinuosa est comme chez les $\mathrm{M}_{2}$.

M1. Le lobe antérieur est oblique par rapport à l'axe antéropostérieur de la dent. Les quatre triangles d'émail sont isolés. Les BRA sont inclinés vers la partie postérieure. LRA 1 s'oriente transversalement à l'axe de la dent ; LRA 2 est incliné vers la partie postérieure.

Le dessin de la linea sinuosa montre quatre grandes échancrures de l'émail qui déterminent les interruptions à la surface occlusale sur les deux côtés du lobe antérieur, à la pointe postérieure de la dent et à la pointe interne du T1. Les premières qui apparaissent sont celles qui se trouvent sur le T1 et le T4 (fig. 3 ).

Il y a deux racines orientées obliquement par rapport à l'axe antéropostérieur de la dent. La racine antérieure a une section plus grande et plus allongée que la postérieure, avec parfois une forme en 8 .

$\mathbf{M}^{2}$. Le lobe antérieur s'oriente obliquement par rapport à l'axe antéropostérieur de la dent chez les formes jeunes, mais il adopte progressivement, avec l'usure, une position transversale. Les triangles d'émail sont pratiquement fermés sur les dents d'individus adultes ; cependant chez les spécimens juvéniles, il y a une légère confluence. Les angles rentrants sont inclinés vers la partie postérieure. Des interruptions d'émail peuvent être observées aux deux angles du lobe antérieur et sur la pointe postérieure du T4.

On observe deux racines, moins inclinées vers l'avant que sur la $\mathrm{M}^{1}$. 

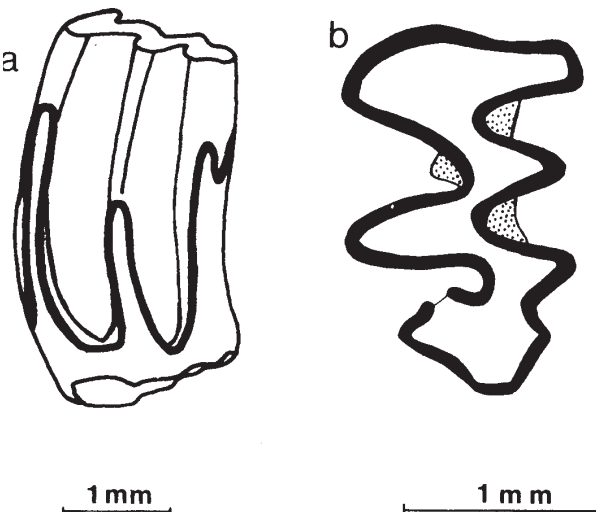

C
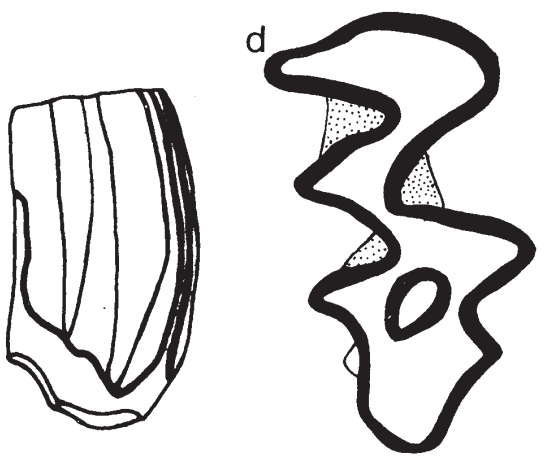

Fig. 10 - Variabilité morphologique dans la $\mathbf{M}^{\mathbf{3}}$ de $M$. medasensis de CSB I. a et b, avec hyposinus développé ; d et c, sans hyposinus.

Morphological variability of the $M$. medasensis $\mathbf{M}^{3}$ from CSB I. a and b, $\mathrm{M}^{3}$ with well developed hyposinus ; $\mathrm{d}$ and $\mathrm{c}$, without hyposinus.

M3. La cuspide postérieure est inclinée vers le côté externe. Sa face interne présente normalement une faible concavité. Sur la face buccale, on observe souvent la crête d'une ébauche de T6 ; cette crête est visible jusqu'à la base de la couronne sur quelques dents, mais couramment, elle disparaît avec l'usure. Le T5 est un triangle bien développé qui se termine par une pointe, mais il n'atteint pas la taille du T2 ou T3. Le puiselet peut être vu sur les dents jeunes, il disparaît lorsque les dents adultes ont déjà des petites racines. Le lobe antérieur et les triangles T2 et T3 ne sont pas complètement isolés, ayant une petite aire de communication entre eux. Deux morphotypes de la linea sinuosa sont distingués. L'un avec un grand développement de l'hyposinus et l'autre, où celui-ci est très faible (fig. 10). Les deux morphotypes sont représentés dans les niveaux $\mathrm{A}$ et $\mathrm{B}$ avec les mêmes proportions : un peu plus de $40 \%$ pour le type sans hyposinus, et presque $60 \%$ pour celui avec un grand hyposinus. Les interruptions d'émail sont présentes au lobe antérieur et sur la cuspide postérieure, où elles apparaissent plus tard.

L'émail est bien différencié, épaissi sur le bord antérieur comme chez les autres dents supérieures.

\begin{tabular}{|lc|c|c|c|c|}
\hline & & $\mathrm{T}$ & $\mathrm{N}$ & $\mathrm{V}$ & $\mathrm{S}$ \\
\hline $\mathrm{M}_{1}$ & $\mathrm{~L}$ & 3.32 & $21 / 37$ & 56 & $* *$ \\
\hline $\mathrm{M}_{2}$ & $\mathrm{~L}$ & 2.00 & $22 / 37$ & 57 & $* *$ \\
\hline $\mathrm{M}_{3}$ & $\mathrm{~L}$ & 1.161 & $22 / 40$ & 62 & - \\
\hline $\mathrm{M}^{1}$ & $\mathrm{~L}$ & 1.604 & $23 / 41$ & 62 & - \\
\hline & $\mathrm{L}$ & 3.419 & $22 / 32$ & 52 & $* *$ \\
\hline $\mathrm{M}^{2}$ & $\mathrm{I}$ & 2.850 & $22 / 32$ & 52 & $* *$ \\
\hline & $\mathrm{L}$ & 2.360 & $23 / 29$ & 50 & $* *$ \\
\hline $\mathrm{M}^{3}$ & $\mathrm{I}$ & 2.096 & $19 / 22$ & 39 & $* *$ \\
\hline
\end{tabular}

Tabl. 5 - Valeurs de significations du test T de Student sur les moyennes des longueurs (L) et largeurs (I) des molaires de $M$. medasensis des niveaux $\mathbf{A}$ et $\mathbf{B}$. ${ }^{* *}$ différence hautement significative. ${ }^{*}$ significative - non significative.

Values and signification of the Student's test $T$ on the length $(\mathrm{L})$ and width $(\mathrm{I})$ means of the $M$. medasensis molars from the levels $\mathbf{A}$ and $\mathbf{B}$. ** highly significative. * significative - not significative difference.

\section{DISCUSSION :}

$M$. medasensis des Iles Medas est très semblable au Mimomys medasensis de notre gisement. A CSB I, nous observons que la taille augmente du niveau $\mathrm{A}$ $\left(\mathrm{M}_{1}=3,23, \mathrm{~N}=21\right)$ jusqu'à niveau $\mathrm{B}\left(\mathrm{M}_{1}=3,42, \mathrm{~N}\right.$ $=37$ ) (fig. 11). Cette augmentation est statistiquement significative surtout chez les $\mathbf{M}_{1}$ et $\mathbf{M}^{1}$ d'après le test $\mathrm{T}$ de Student (tabl. 5). Il y a aussi une augmentation 
de l'hypsodonte maximale (hauteur/longueur), mesurée sur la $\mathrm{M}_{1}$, qui passe de 1,53 dans le niveau $\mathrm{A}$ à 1,64 dans le niveau B. M. medasensis des Iles Medas présente une taille des $\mathrm{M}_{1}\left(\mathrm{LM}_{1}=3,32, \mathrm{~N}=16\right.$; Michaux 1971) qui s'intercale entre celles des niveaux A et B de CSB I ; cependant l'hypsodontie maximale
$(1,5)$ est semblable à celle de $M$. medasensis du niveau A de CSB I. M. medasensis a été aussi retrouvé dans des gisements sédimentaires du bassin du Júcar, Province d'Albacete (Mein, Moissenet \& Truc 1978 ; Aguirre et alii 1982 ; Alberdi et alii 1983) dans la coupe de Valdeganga (niveau I et II) et à El Rincón 1 .

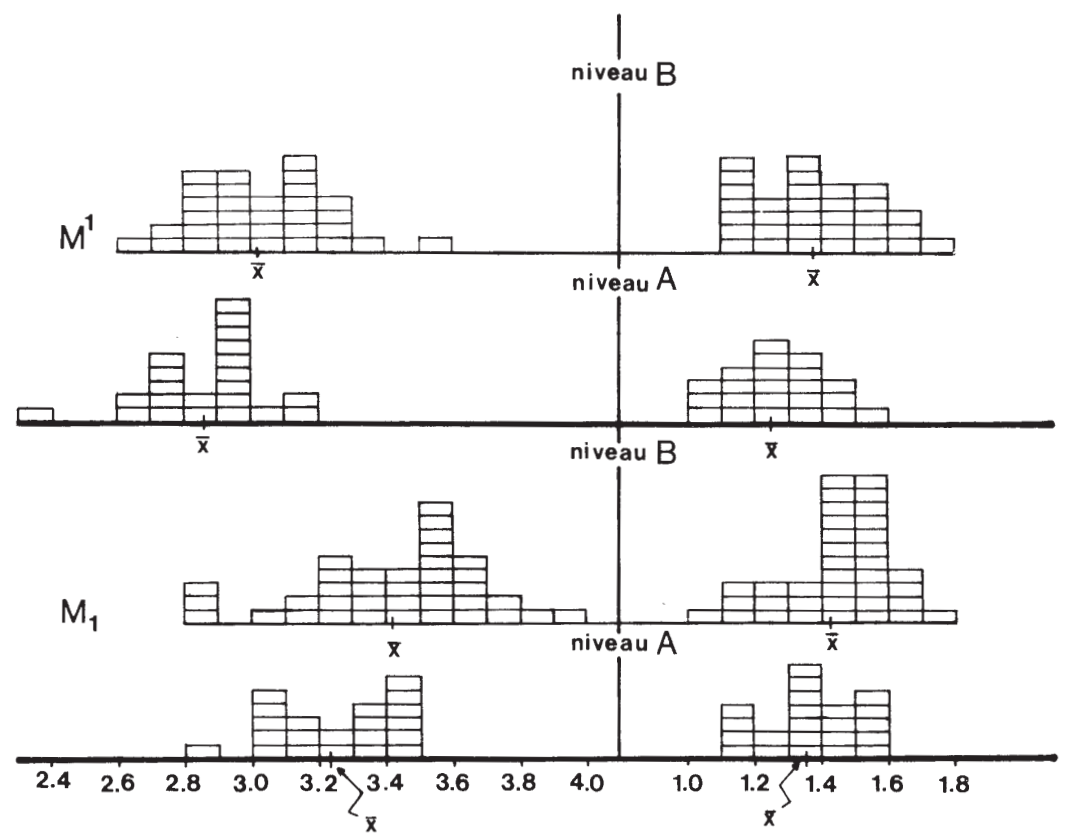

Fig. 11 - Histogramme de la longueur et de la largeur des premières molaires de $M$. meáasensis des populations des niveaux superposés A et B de CSB I. En haut $\mathrm{M}^{1}$, en bas $\mathrm{M}_{1}$. Voir tableau 5 sur la signification des différences statistiques.

Length and width histograms of the $M$. medasensis first molars from the two populations of the overling levels A and B from CSB I. Below, $\mathrm{M}_{1}$; above, $\mathrm{M}^{1}$. See table 5 on the signification of the statistical differences.

$M$. medasensis est une espèce ibérique semblable à $M$. pliocaenicus et à $M$. ostramosensis, mais différant par le nombre de racines de la $\mathrm{M}^{1}$. M. pliocaenicus a 3 racines isolées ; $M$. ostramosensis a soudé partiellement ses deux racines antérieures, alors que $M$. medasensis en a seulement 2 , dont la racine antérieure peut être bilobée et parfois légèrement étranglée. Cependant ce caractère montre une variabilité intrapopulationnelle importante, par exemple chez $M$. tornensis, et ne peut suffire à établir une diagnose.

La taille de $M$. medasensis du niveau A est semblable à celle de $M$. pliocaenicus, alors qu'au niveau B elle devient aussi grande que $M$. ostramosensis.
Une autre différence remarquée entre $M$. medasensis et $M$. pliocaenicus d'après Janossy \& Van der Meulen (1975) serait la disparition plus précoce du puiselet sur les dents de $M$. medasensis. Cependant, quelques populations attribuées soit à $M$. pliocaenicus soit à $M$. ostramosensis, comme celle de Kadzielnia (Chaline 1974, fig. 11 ; Chaline \& Laurin 1986 ; Van der Meulen com. pers.) ont des dents très hypsodontes qui ont perdu déjà le puiselet tout comme chez $M$. medasensis. Il est donc possible que ces espèces puissent être réunies dans le futur, sur la base d'observations plus nombreuses. 
Mimomys rex KORMOS, 1934

1934 - M. rex n.sp. : KoRMOS, p. 24-25, fig. 49.

1954 - Kislangia ondatrina n.g. n.sp. : KRETZOI, p. 247.

1965 - Mimomys (Kislangia) sp. : Alexandrova, fig. 12.

LOCALITÉ TYPE :

Villany 3 (Hongrie).

DIAGNOSE :

Mimomys de grande taille, (longueur des $\mathrm{M}_{1}=\mathbf{3}$, $8-4,2$ ), caractérisé par la réduction de la cuspide antérieure des $\mathbf{M}_{1}$, l'absence du prismenfalte et du mimomyskante, et par un inselfalte très profond. Le cément est très abondant (d'après la description de Kormos 1934, p. 24-25).

DISTRIBUTION :

Hongrie (Villany, Kislang), Ukraine (Kotlovina), ?France et Espagne.

\section{Mimomys aff. rex}

texte-fig. 12 ; pl. 3

?1974 - Mimomys cf. rex Kormos : Chaline \& Michaux, p. 55-83, pl. 4, fig. 1-4, 7-11 et 13 20 ; pl. 7, fig. 9-12, pl. 8, fig. 1-7.

1978 - Mimomys rex Kormos : Mein, Moissenet \& Truc, p. 112-122, fig. 7 a-g.

?1984 - Mimomys clairi nov. sp. : Chaline, p. 255 fig. 10 .

\section{MATÉRIEL DE CSB I :}

Niveau $A: 4 M_{1}, 3 M_{2}, 1 M_{3} ; 1 M^{1}$ et $1 M^{3}$

Niveau B : 7 mandibules inférieures, $15 \mathrm{M}_{1}, 13 \mathrm{M}_{2}$, $11 \mathrm{M}_{3} ; 7 \mathrm{M}^{1}, 11 \mathrm{M}^{2}$ et $7 \mathrm{M}^{3}$.

Niveau D : $1 \mathrm{M}^{1}, 2 \mathrm{M}^{2}$ et $2 \mathrm{M}^{3}$.

MENSURATIONS :

\begin{tabular}{|c|c|c|c|c|c|c|c|c|}
\hline & \multicolumn{4}{|c|}{ L } & \multicolumn{4}{|c|}{1} \\
\hline & & Min. & Moy. & Max. & $\mathrm{N}$ & Min. & Moy. & Max. \\
\hline \multirow{3}{*}{ 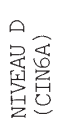 } & $M^{1}$ & & 2.55 & & 1 & & 1.73 & \\
\hline & $\mathrm{M}^{2}$ & 2.76 & 2.78 & 2.80 & 2 & 1.73 & 1.73 & 1.73 \\
\hline & $\mathrm{M}^{3}$ & 2.68 & 2.68 & 2.68 & 2 & 1.54 & 1.56 & 1.58 \\
\hline \multirow{6}{*}{ 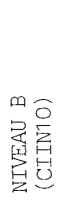 } & $\mathrm{M}_{1}$ & 3.39 & 3.91 & 4.70 & 22 & 1.48 & 1.77 & 2.13 \\
\hline & $M_{2}^{\prime}$ & 2.41 & 2.63 & 3.00 & $18 / 19$ & 1.46 & 1.63 & 2.01 \\
\hline & $\mathrm{M}_{3}$ & 2.17 & 2.42 & 2.64 & 13 & 1.18 & 1.34 & 1.58 \\
\hline & $M_{1}^{1}$ & 3.20 & 3.53 & 3.91 & 7 & 1.71 & 1.92 & 2.05 \\
\hline & $M^{2}$ & 2.64 & 2.85 & 3.00 & 11 & 1.62 & 1.85 & 2.09 \\
\hline & $\mathrm{M}^{3}$ & 2.60 & 2.70 & 2.84 & 7 & 1.38 & 1.57 & 1.73 \\
\hline \multirow{5}{*}{ 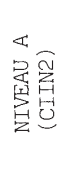 } & $M_{1}$ & 4.03 & 4.21 & 4.46 & 4 & 1.77 & 1.93 & 2.05 \\
\hline & $\mathrm{M}_{2}$ & 2.60 & 2.74 & 2.92 & 3 & 1.54 & 1.63 & 1.77 \\
\hline & $\mathrm{M}_{3}$ & & 2.41 & & 1 & & 1.30 & \\
\hline & $\mathrm{M}^{1}$ & & 3.55 & & 1 & & 2.30 & \\
\hline & $\mathrm{M}^{3}$ & & 2.35 & & 1 & & 1.25 & \\
\hline
\end{tabular}

Tabl. 5 - Mesures de la longueur $(L)$ et de la largeur (I) des dents de $M$. aff. rex du CSB I, niveaux $\mathbf{A}, B$ et $\mathbf{D}$.

Measurements of the length $(L)$ and width (I) of the $M$. aff. rex from CSB I, levels $A, B$ and $D$. 


\section{DESCRIPTION :}

Toutes les dents ont deux racines sauf les $\mathrm{M}^{1}$ qui en ont trois. Le cément est très abondant. L'émail est différencié chez les dents adultes.

M1. Dans le complexe antéroconide, le puiselet peut se voir encore dans des stades d'usure assez avancés, même chez des dents avec des racines séparées et bien développées. Le LRA 4 est plus profond que l'inselfalte. Les triangles d'émail linguaux sont plus développés que les buccaux, ce qui entraîne la dissymétrie du dessin de la surface occlusale. Tous les triangles d'émail sont presque fermés à l'exception de T1 et T2 qui communiquent entre eux ; cet espace se réduit et disparaît chez les dents très usées (fig. 12). Les synclinaux linguaux s'orientent transversalement à l'axe de la dent, ayant leurs pointes légèrement recourbées vers la partie antérieure. Les BRA sont fortement recourbés vers l'avant.

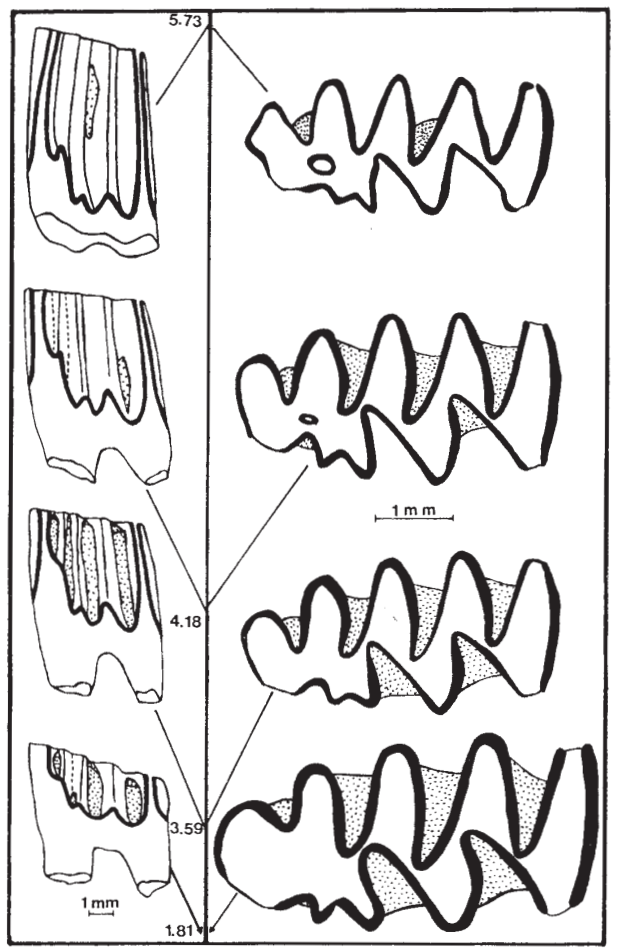

Fig. 12 - Séquence d'usure de la $\mathbf{M}_{1}$ chez $M$. aff. rex de CSB $\mathbf{I}$. Wear sequence of $M$. aff. rex from CSB I.
Des interruptions d'émail s'observent sur la surface occlusale aux côtés interne et externe du lobe postérieur, ainsi qu'à la partie antéro-externe de la cuspide antérieure. Dans la linea sinuosa (fig. 13) l'antérosinuide est presque perpendiculaire dès la surface occlusale et ceci jusqu'à la base, ce qui donne une interruption relativement petite sur le lobe antérieur.

M2. T1 et T2 communiquent, T2 et T3 sont pratiquement isolés. Les angles rentrants internes s'orientent perpendiculairement à l'axe longitudinal de la dent, leurs pointes étant légèrement recourbées vers l'avant ; les angles externes s'orientent vers l'avant. La linea sinuosa (fig. 13) détermine trois grandes échancrures: l'antérosinuide, l'hyposinulide et l'hyposinuide.

M3. T1 et T2 confluent, étant pratiquement opposés chez les dents très usées. L.es angles rentrants internes sont plus développés que les externes; LRA 2 s'oriente perpendiculairement à l'axe longitudinal de la dent ; LRA 1 est moins profond que LRA 2 et sa pointe s'incline vers la partie antérieure. Le BRA 1, plus ouvert et développé que le BRA 2, s'oriente perpendiculairement à l'axe, quelquefois légèrement incliné vers l'avant. Le BRA 2 s'incline aussi vers l'avant. Le dessin de la linea sinuosa est semblable à celui de la $\mathrm{M}_{2}$.

M1. Le lobe antérieur est incliné par rapport à l'axe longitudinal de la dent. Les quatre triangles d'émail sont isolés. Le développement des angles rentrants est inégal, les buccaux étant plus profonds que les linguaux, ce qui entraîne une petite dissymétrie du dessin dentaire. Les angles rentrants buccaux sont plus inclinés, par rapport à l'axe antéro-postérieur, que les linguaux qui sont transversaux. Les distosinus, le protosinus, l'antérosinus et l'antérosinule sont très hauts (fig. 13) et ils sont responsables des interruptions de l'émail sur la surface occlusale. La dent développe trois racines, une antérieure robuste avec une section à peu près arrondie, une postérieure moins développée et, enfin, une autre petite entre les deux sur le côté interne.

$\mathbf{M}^{2}$. Les trois angles d'émail confluent chez les dents issues d'individus très jeunes. Le LRA 2 est un angle très profond qui oriente sa pointe fortement vers l'arrière comme le BRA 2. Le BRA 1 a une orientation plus transversale. Les interruptions d'émail peuvent être observées sur les deux côtés du lobe antérieur et à la pointe postérieure du T4.

$\mathbf{M}^{3}$. Le sommet postérieur ne présente pas de forme hémisphérique puisqu'il se trouve comprimé sur le côté lingual. Le puiselet a une forme de rectangle 


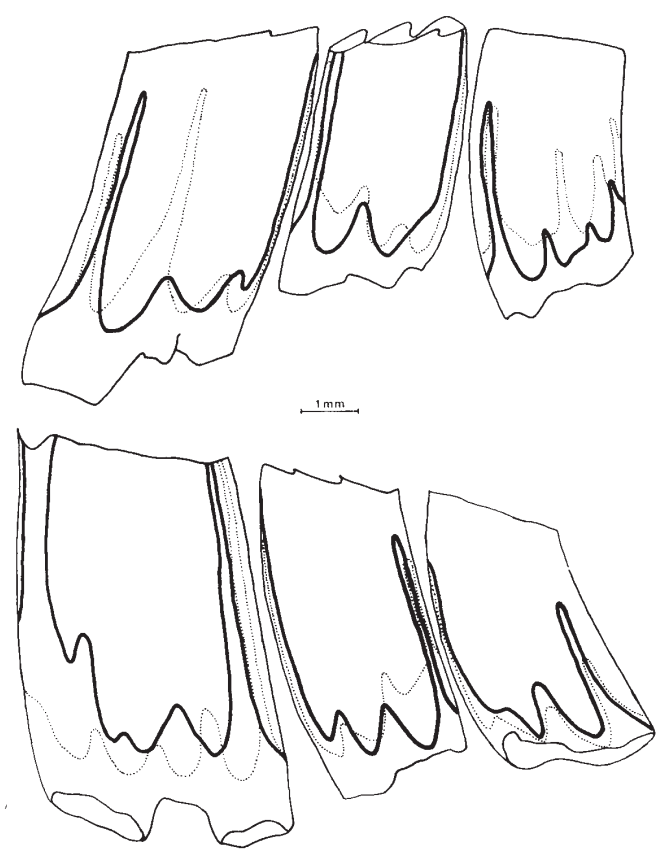

Fig. 13 - Linea sinuosa chez $\boldsymbol{M}$. aff. rex de CSB I. En haut, molaires supérieures; en bas, molaires inférieures.

Linea sinuosa of $M$. aff. rex from CSB I. Above, upper molars ; below, lower molars.

étroit et allongé dont l'axe longitudinal forme un angle de $45^{\circ}$ environ avec celui de la dent. Cette structure peut être observée sur les dents très usées, le puiselet étant très profond. Une ébauche de T6 s'observe sur quelques dents. T2 et T3 communiquent peu dans le cas de dents issues d'adultes et ils s'isolent avec l'usure ; le degré de confluence est moindre que celui du T2 avec le lobe antérieur. Le BRA 2 est profond et sa pointe est fortement recourbée vers l'arrière. Le BRA 3, moins profond que les autres angles buccaux, est cependant bien marqué.

La linea sinuosa montre un hiposinus bien développé. La cuspide postérieure présente une échancrure haute, le distosinus, avec, de chaque côté, une autre échancrure plus petite (fig. 13).

\section{DISCUSSION :}

$M$. aff. rex ressemble à $M$. medasensis, même dans la $\mathrm{M}_{1}$ et $\mathrm{M}^{3}$; c'est à cause de cela que sa différenciation a été très difficile, surtout dans le niveau B. Sur la figure 14 on peut voir la distribution L/l (longueur /largeur) des $\mathbf{M}_{1}$ de ces espèces dans le niveau cité. Cependant on a trouvé quelques différences qui nous permettent de les séparer ;

$\mathbf{M}_{1}$ : 1) Chez $M$. aff. rex le dessin de la surface occlusale est plus comprimé dans le sens antéro- postérieur, ce qui donne aux dents un aspect plus robuste que chez $M$. medasensis.

2) Chez $M$. aff. rex, le puiselet peut être présent même sur des dents usées aux racines séparées et bien développées, tandis que chez $M$. medasensis, le puiselet disparaît avec le début de l'usure.

3) Les deux espèces montrent, sur la face occlusale, des interruptions d'émail dans la partie antéroexterne. Cependant, ces interruptions sont plus grandes chez $M$. medasensis que chez $M$. aff. rex. L'antérosinuide descend presque verticalement sur les $M_{1}$ de $M$. aff. rex tandis que sur celles de $M$. medasensis il descend obliquement, laissant une plus grande surface dépourvue d'émail.

4) Le mimosinuide est normalement absent ou très peu développé chez $M$. aff. rex, tandis que chez $M$. medasensis il atteint toujours un grand développement.

$\mathbf{M}^{1}$ : Les $\mathrm{M}^{1}$ de $M$. aff. rex présentent trois racines, tandis que celles de $M$. medasensis en possèdent seulement deux. Les échancrures de la linea sinuosa sont moins développées chez $M$. aff. rex, où les interruptions d'émail apparaissent sur la surface occlusale avec des stades d'usure plus avancés que chez $M$. medasensis. 
$\mathbf{M}^{3}$ : Sur les $\mathrm{M}^{3}$, les BRA sont légèrement plus ouverts chez $M$. medasensis que chez $M$. aff. rex, surtout le BRA 2 qui, couramment n'est pas recourbé vers l'avant, au contraire de ce qui s'observe chez $M$. aff. rex. La cuspide postérieure est plus aplatie chez $M$. aff. rex que chez $M$. medasensis, où la section est plus proche d'un triangle. Sur le premier, le puiselet est généralement rectangulaire sur les dents adultes, tandis que chez $M$. medasensis il a une forme ovale ou circulaire ; le puiselet est plus fugace chez $M$. medasensis. $M$. aff. rex a des triangles moins dissymétriques que $M$. medasensis. Le BRA 2 est plus profond chez le premier que chez le second, avec la pointe plus fortement recourbée vers l'arrière.
La grande taille - la plus grande rencontrée chez les Mimomys - , l'aspect comprimé des dents dans le sens antéro-postérieur - ce qui se traduit par une plus grande largeur relative - , le grand développement du cément, le puiselet relativement persistant et la très petite aire dépourvue d'émail sur le lobe antérieur de la $\mathrm{M}_{1}$ sont autant de caractères qui rapprochent la population de CSB I de $M$. rex de Villany 3. Cette espèce a été décrite par Kormos (1934) avec très peu de matériel et elle est extrêmement rare en Europe Centrale. Elle a été trouvée aussi à Villany 5 et Kislang et Krezoi (1954) en a fait un sous-genre de Mimomys (Kislangia) auquel Rabeder (1981) ajoute deux nouvelles espèces, $M$. (Kislangia) regulus et $M$. (Kislangia) praerex, de taille plus petite.

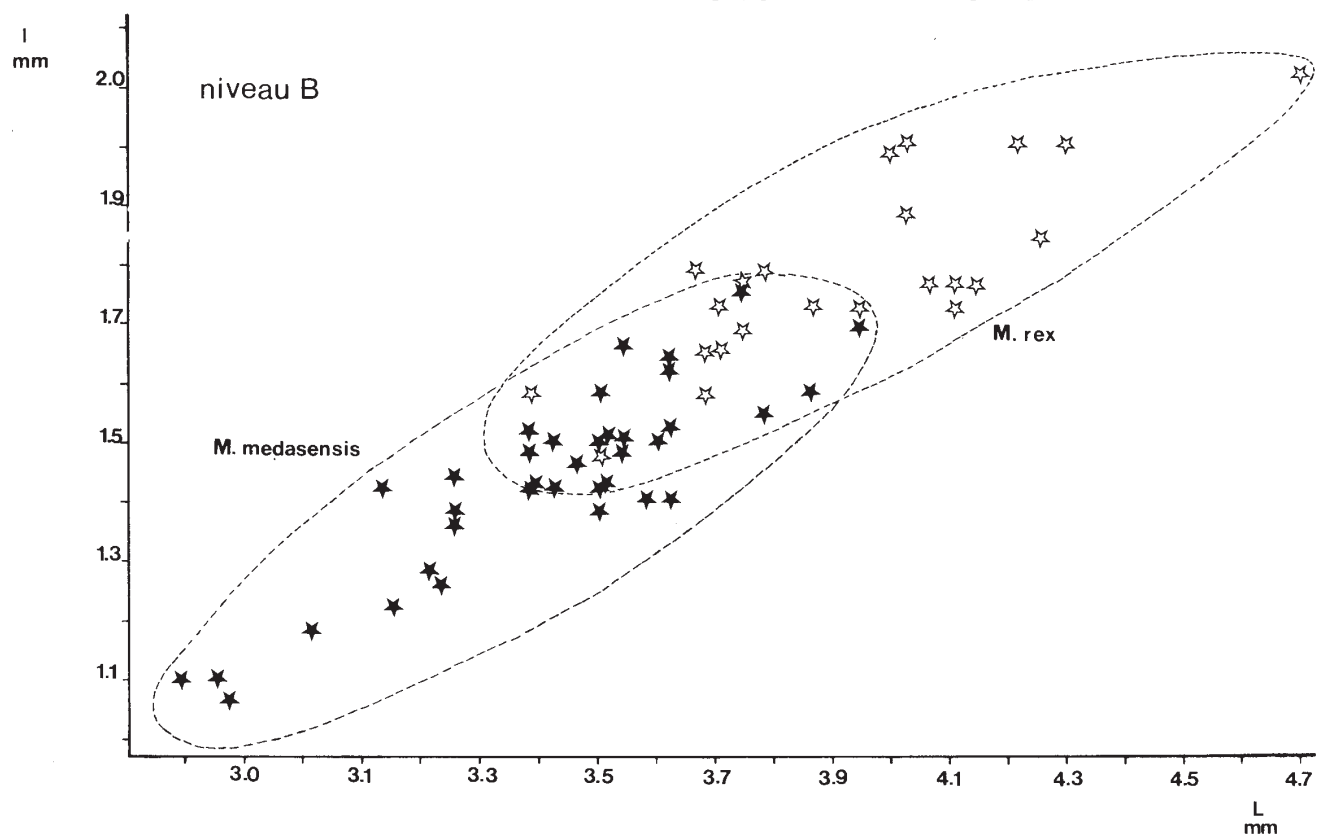

Fig. 14 - Diagramme de dispersion longueur (L)/largeur (I) des $\mathbf{M}_{1}$ de $M$. medasensis et $M$. aff. rex du niveau B de CSB I. Scatter diagram length (L)/width (l) of the $M$. medasensis and $M$. aff. rex $\mathrm{M}_{1}$ from CSB I, level B.

En Europe occidentale cette espèce est beaucoup plus fréquente. Elle a été citée dans le remplissage bressan en France (gisements de Levernois et Montagny, Chaline \& Michaux 1974 ; Chaline 1984) et dans le bassin du Júcar en Espagne (Mein et alii 1978). Ces populations occidentales, comme celle de CSB I, diffèrent des populations orientales par un ACC plus primitif aux $\mathrm{M}_{1}$ car ils conservent mimomyskante et prismen- falte alors que ces structures n'existent pas sur les dents adultes de la population type de Villany 3.

Il y a aussi des différences très nettes entre les populations espagnoles et françaises. Le cément est plus abondwnt sur toutes les dents de la population de CSB I. Sur le lobe antérieur de la $\mathrm{M}_{1}$, le prismenfalte est beaucoup plus large et profond dans les populations françaises, et le T4 est plus développé. Par contre, 
dans les populations espagnoles, l'inselfalte est très profond, même plus que le prismenfalte, et le mimomyskante est plus proche du BSA 3 que dans les formes du Jura. Il y a 3 racines aux $\mathrm{M}^{2}$ dans les populations françaises (Chaline \& Michaux 1974, fig. 20, p. 73) et deux sur toutes celles de CBS I.

Tout cela nous amène à désigner sous le nom de $M$. aff. rex notre population de CBS I. Il est possible qu'elle puisse être une race géographique de $M$. rex, mais il est aussi possible que les différents groupes de ces grands Mimomys n'appartiennent pas à la même lignée, étant donné qu'en Espagne, de grands Mimomys existent ( $M$. aff. cappettai, Van der Weerd 1976) depuis le Villanyen inférieur, pouvant être à l'origine de $M$. aff. rex. Par contre, en Europe centrale, des espèces plus petites [M. (Kislangia) regulus, $M$. (Kislangia) praerex RABEDER, 1981] ont été proposées comme ancêtres de $M$. (Kislangia) rex.

\section{ULTRASTRUCTURE DE L'ÉMAIL}

Koenigswald (1982) a distingué trois types d'émail dans les molaires d'Arvicolidés (pl. 4) : radial, tangentiel et lamellaire. D'après cet auteur, l'émail primitif est le radial, puis apparaît l'émail tangentiel et finalement le type lamellaire.

A CSB I, les trois espèces d'Arvicolidés ne présentent pas de différences significatives dans la structure de leur émail (fig. 15 et pl. 4). Il s'agit d'un émail hautement différencié et tous les triangles de la dent ont la même structure. Côté convexe, il y a un émail tangentiel et un émail radial. Le premier se développe dans la moitié externe de la bande d'émail et atteint les marges externe et interne du triangle ; ceci est un trait caractéristique des formes avec un haut degré de différenciation. L'émail radial se développe sur la moitié interne de la bande. Côté concave, l'émail radial est présent à la partie externe de la bande d'émail, et l'émail lamellaire, dans la partie interne. Celui-ci occupe toute la longueur de la bande d'émail et il atteint le bord interne ; l'émail radial est absent entre l'émail lamellaire et le bord interne, au contraire de ce qui peut être observé chez d'autres formes moins évoluées.

Les populations de Mimomys de CSB I présentent donc une structure de l'émail totalement évoluée, d'après la polarité évolutive proposée par Koenigswald (1980) qui caractérise les espèces les plus récentes du genre.

\section{RÉSULTATS ET CONCLUSIONS}

CSB I est le premier gisement du Villanyen récent d'Espagne qui possède trois espèces d'Arvicolidés dans une séquence stratigraphique régulière. Les populations sont très riches et les conditions taphonomiques sont comparables d'un niveau à l'autre.

L'étude de la variabilité à l'intérieur des populations et entre des populations successives, effectuées sur des échantillons importants, a permis d'observer des différences métriques et morphologiques plus importantes entre des individus jeunes et adultes d'une espèce de Mimomys, qu'entre des adultes d'espèces différentes. Ceci est en partie la raison d'une certaine confusion dans la systématique de ce genre qui est à la base de la chronologie du Pliocène et du Pléistocène inférieur continentaux.
Etant donnée la différenciation de notre région ibéroccitane (Thaler 1966 ; Cabrera et alii 1983), où la majorité des micromammifères sont endémiques déjà depuis le Pliocène inférieur, seules quelques espèces, dont les Arvicolidés, permettent la corrélation interrégionale. La comparaison entre nos populations et celles d'autres régions porte sur de nombreux caractères.

M. medasensis pourrait être un Arvicolidé endémique, mais il semble possible de le rapprocher des espèces $M$. pliocaenicus et $M$. ostramosensis. Le nombre de racines de $M^{1}$ le sépare de ces 2 espèces mais ce caractère a certainement une variabilité instraspécifique. $M$. medasensis augmente sa taille et son hypsodontie dans la succession stratigraphique de CSB I de façon statistiquement significative. 


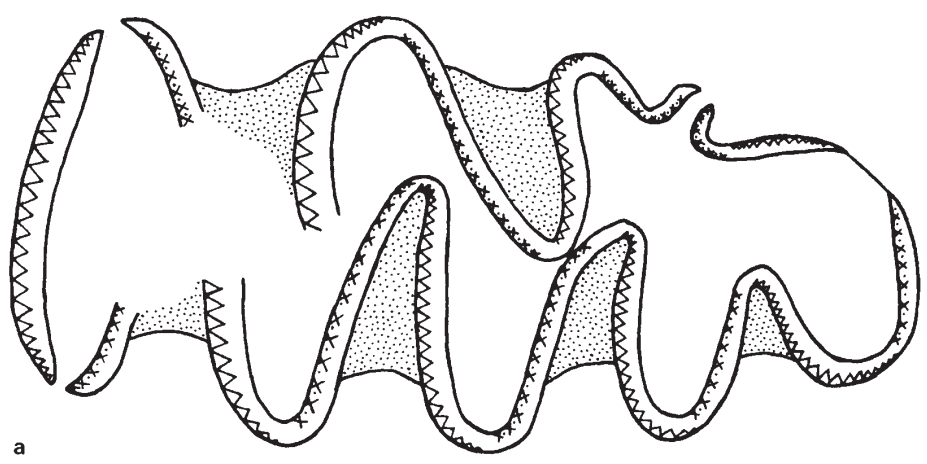

b

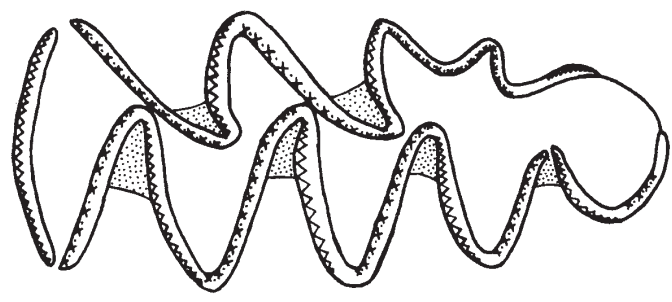

c
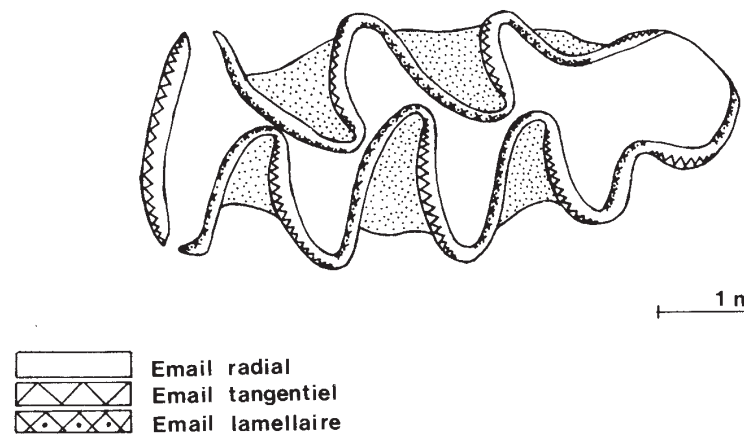

Fig. 15 - Modèle de l'émail dans la $\mathbf{M}_{1}$ chez $\boldsymbol{M}$. aff. rex (a), $\boldsymbol{M}$. medasensis (b) et $M$. tornensis (c). En blanc, émail radial ; triangles, émail tangentiel ; et croix et points, émail en chevrons (lamellaire).

M1 enamel pattern of $M$. aff. rex (a), M. medasensis (b) et $M$. tornensis (c). White, radial enamel ; triangles, tangential enamel ; and crosses and points, lamellar enamel. 
Le cas de $M$. aff. rex est aussi particulier, car ce n'est pas tout à fait sûr qu'il appartienne à l'espèce d'Europe centrale. Des différences géographiques existent entre ses populations. Plusieurs auteurs admettent que $M$. rex a pu dériver de $M$. cappettai MichauX (p. ex. Michaux 1971 ; Chaline \& Michaux 1974 ; Rabeder 1981). M. aff. rex de CSB I et des autres gisements espagnols montre une morphologie plus proche de l'ancêtre hypothétique, mais une taille et une hypsodontie très développées, semblables à $M$. cf. rex (M. clairi) de Bresse et à $M$. rex de Villany 3 .

$M$. tornensis est donc la seule espèce de CSB I qui puisse être rapportée à une espèce connue en Europe centrale. La plupart des autres micromammifères de CBS I (Stephanomys, Castillomys, Desmana inflata, Apodemus occitanus, Prolagus calpensis, etc.) sont endémiques de la région ibéroccitane. C'est la première fois que $M$. tornensis a été reconnu dans la Péninsule Ibérique. La comparaison détaillée de notre population avec celles d'autres gisements du centre de l'Europe a apporté d'importants résultats qui affectent même la diagnose de l'espèce. Les populations de M. tornensis d'Osztramos-3 (type), de la Tchécoslovaquie et de CSB I se ressemblent beaucoup ; elles montrent toujours un mimomyskante à la $\mathrm{M}_{1}$ dans des proportions qui varient selon l'âge des individus. Le BSA 4 indiqué dans la diagnose est en réalité le mimomyskante situé en position avancée, par suite de la réduction de l'inselfalte qui fait souvent défaut.

L'établissement d'une biochronologie à partir de ces faunes présente de grandes difficultés, car il y a peu de successions biostratigraphiques sur lesquelles elle puisse être fondée. Le Villafranchien serait la seule unité chronostratigraphique continentale qui couvrirait cette période. Il a été défini par Pareto (1865) à Villafranca d'Asti (Piémont, Italie), et caractérisé surtout par des faunes de grands mammifères (Anancus, E. (M.) meridionalis, Tapirus, Equus stenonis, Leptobos).

Dans la localité type du Villafranchien, à Arondelli, 6 espèces de rongeurs, dont 2 espèces de Mimomys (M. polonicus ou $M$. cf. stehlini et $M$. (C.) gracilis) ont été reconnues (Michaux 1970). Cette association a été corrélée avec les gisements de Hajnacka et Seynes et située dans l'unité MN 16 de l'échelle biochronologique de Mein (1975). L'unité suivante (MN 17), dernière de cette échelle, est caractérisée par l'Arvicolidé $M$. pliocaenicus, et pourrait correspondre au Pliocène ou en partie déjà au Pléistocène.
Le Villafranchien a été reconnu aussi dans la région de Val d'Arno (Toscana, Italie). Plusieurs auteurs ont étendu la définition du Villafranchien aux niveaux stratigraphiques supérieurs de ce bassin où se trouve la localité-type de $M$. pliocaenicus. Azzarolli (1970) inclut aussi dans le Villafranchien les faunes à Microtus (Allophaiomys). Fejfar \& Heinrich (1981) réduisent au contraire le Villafranchien aux faunes de la région-type.

Le Villafranchien étant insuffisamment caractérisé la plupart des auteurs préfèrent utiliser les unités biochronologiques (âges mammaliens) Villanyen et Biharien, tels qu'ils ont été redéfinis par Kretzoi en 1969 (Michaux 1970 ; Van der Meulen 1973 ; Benda et alii 1977 ; Van der Meulen 1978).

D'après Kretzoi (1969), le Villanyen comprendrait deux périodes : le Villanyen ancien (Beremendien), caractérisé par la prédominance de Dolomys, et le Villanyen récent (Arnien ou Kislangien) où le genre Mimomys est dominant. Le Biharien, caractérisé par la dominance des Arvicolidés arrizodontes (Arvicola, Microtus) comprendrait trois périodes, dont la plus ancienne est définie par la dominance d'Allophaiomys.

Le Villanyen récent à Mimomys pliocaenicus est daté d'environ 1,9 Ma., et le Biharien ancien à Microtus (Allophaiomys) pliocaenicus à 1,2 Ma. (Van der Meulen 1978). Dans l'intervalle où se trouve la limite entre ces deux périodes, se situe aussi la limite Plio/Pléistocène placée à 1,8 ou à 1,6 Ma. selon les auteurs (Berggren \& Van Couvering 1974 ; Tauxe et alii 1983). Dans cet intervalle se situent des faunes où Mimomys est dominant et où parfois un primitif Allophaiomys nommé $A$. deucalion est présent dans des faibles proportions (voir tableau 7).

Plusieurs auteurs ont adopté une définition différente du Biharien, sur le critère de première apparition d'Allophaiomys (p.ex. Fajfar \& Heinrich 1981, 1983). Ils ont alors classé les faunes de cet intervalle selon la présence ou l'absence d' $A$. deucalion. Cela entraîne cependant une succession chaotique des espèces de Mimomys. Notre proposition biochronologique (tabl. 7) montre par contre une succession ordonnée des espèces de Mimomys. $M$. tornensis et $M$. ostramosensis remplacent $M$. newtoni et $M$. pliocaenicus, puis $M$. reidi est remplacé par $M$. pusillus. La présence ou l'absence d'Allophaiomys deucalion ne sont pas ici considérées comme un critère biochronologique. Ses faibles fréquences amènent à tenir compte des effets taphonomiques et d'échantillonage. 
La présence de $M$. tornensis permet de corréler la faune de CSB I avec les faunes de cet intervalle, comme celles d'Osztramos 3 ou Kolinany. Ces faunes appartiennent au Villanyen récent dans son acception originelle, et se placent autour de la limite Pliocène/Pléistocène.

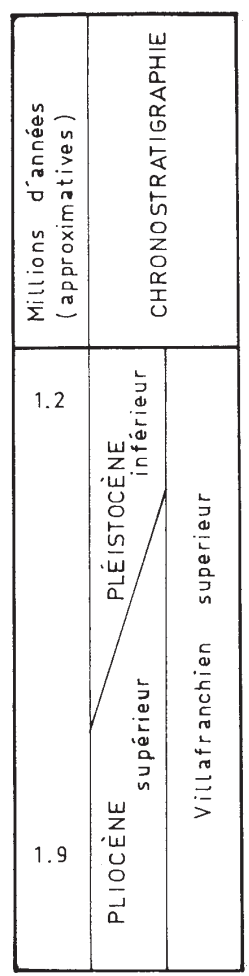

\begin{tabular}{|c|c|c|c|c|c|c|c|c|c|c|c|c|c|c|c|c|}
\hline \multicolumn{2}{|c|}{$\begin{array}{c}\text { Ages } \\
\text { mammaliens }\end{array}$} & \multirow[b]{2}{*}{$A \times A$} & \multirow[b]{2}{*}{ 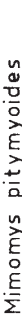 } & \multirow[b]{2}{*}{ 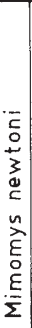 } & \multirow[b]{2}{*}{$\begin{array}{l}\frac{n}{n} \\
c \\
d \\
\frac{1}{2} \\
o \\
+ \\
\Sigma\end{array}$} & \multirow[b]{2}{*}{ 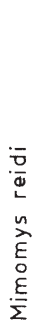 } & \multirow[b]{2}{*}{$\stackrel{n}{\Xi}$} & \multirow[b]{2}{*}{ 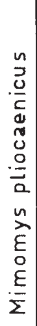 } & \multirow[b]{2}{*}{ 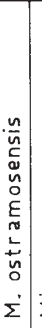 } & \multirow{2}{*}{\multicolumn{2}{|c|}{ 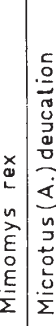 }} & \multirow[b]{2}{*}{ 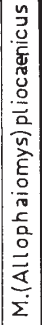 } & \multicolumn{4}{|c|}{ BIOCHRONOZONES } \\
\hline 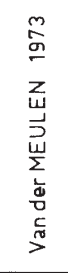 & 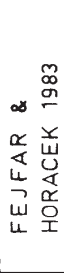 & & & & & & & & & & & & 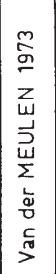 & $\begin{array}{l}\frac{n}{\sigma} \\
\frac{\pi}{\sigma} \\
\frac{z}{\Sigma}\end{array}$ & 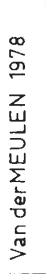 & 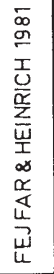 \\
\hline 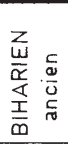 & & $\begin{array}{l}\text { DEUTCH-ALTENBURG } \\
2 \mathrm{C1} \\
\text { VCELARE } 3 \mathrm{~B} 1\end{array}$ & & & + & & + & & + & & & + & 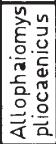 & & 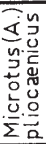 & 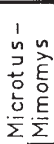 \\
\hline c & 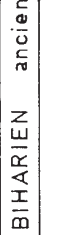 & $\begin{array}{l}\text { OSZTRAMOS } 3 \\
\text { VCELARE } 3 \\
\text { KOLINANY } 3 \\
\text { PLESIVEC } \\
\text { KOLINANY } 1,2 \\
\text { KADZIELNIA }\end{array}$ & + & & + & + & & ? & + & & + & & & & 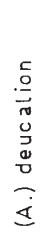 & \\
\hline$z$ & & VILLANY 5 & & + & & + & & + & & + & + & & 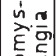 & & $\Sigma$ & \\
\hline $\begin{array}{l}\frac{2}{2} \\
\vdots \\
\\
\\
>\end{array}$ & $\begin{array}{l}z \\
w \\
z \\
z \\
d \\
\pm \\
y \\
z\end{array}$ & $\begin{array}{l}\text { KISLANG } \\
\text { VILLANY } 3 \\
\text { TEGELEN }\end{array}$ & & + & & + & & + & & + & & & 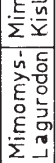 & $z_{\Sigma}$ & 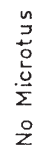 & 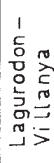 \\
\hline
\end{tabular}

Tabl. 7 - Séquence biochronologique et corrélation chronostratigraphique proposées pour la période Villanyen récent - Biharien ancien. Les gisements proches de la limite Plio-Pléistocène sont rangés très différemment par les auteurs, qui suivent le critère de présence/absence d'Allophaiomys. Notre proposition offre une meilleure distribution des espèces de Mimhmys. Le gisement espagnol de CSB I peut être situé dans cet intervalle.

Biochronological sequence and chronostratigraphic correlation proposed for the late Villanyan-Early Biharian period. The sites near the Plio-Pleistocene boundary are arranged in different ways by authors following the criterion of the Allophaiomys presence/absence. Our proposition offers a better distribution of the Mimomys species. The spanish site CSB I can be situated in this interval.

\section{Remerciements}

Nous sommes très reconnaissants au Dr. Alber Van der Meulen de l'Université d'Utrecht, qui a bien voulu discuter nos résultats et nous fournir du matériel de comparaison de Tegelen. Les Drs. Sesé et Ruiz Bustos, de Madrid, nous ont aussi aimablement permis de consulter leurs collections. Les
Drs. J. Chaline, P. Mein et J. Michaux sont vivement remerciés pour la révision critique du manuscrit, et J. Michaux et B. Bachelet aussi par leur obligeance dans la correction du texte français. Les dessins sont de l'un des auteurs (J.E.A.), la dactylographie est due à $\mathrm{Mms}$ M. de Andrés et I. Corchón. 


\section{RÉFÉRENCES BIBLIOGRAPHIQUES}

Aguirre E., Arias C., Bonadonna A., Civis J., DABRIO C., GOY J.L., LOPEZ N., MORAlES J., PEREZ GonZalez A., PORTA J. \& ZAZO C. (1982) Plio-Pleistocene transition in the Iberian Peninsula. IGCP Projet 41. Final Report (Moscow).

AGUSTI J. \& GALOBART A. (1986) - La sucesión de micromamiferos en el complejo cárstico de Casablanca (Almenara, Castellón) : Problemática biogeográfica. II Jornadas de Paleontologia, Abstract, Sabadell : 48.

AlBERTI M.T., ARIAS C., BIGAZZI A., BONADONNA A. LEONE S., LOPEZ N., MICHAUX J., MORALES J., ROBLES F. \& SORIA M.D. (1982) - Nuevo yacimiento de Moluscos y Vertebrados del Villafranquiense de la cuenca del Júcar (Albacete, Espana). Coll. « Le Villafranchien Méditerranéen », Lille : 255-271.

ALEXANDROVA L.-P. (1965) - Fossil Eopleistocene Voles (Rodentia, Microtinae) of Southern Moldavia and South-Western Ukraine. In : « Stratigraphic importance of small mammalian antropogene fauna ». Edit. Nauka, Moscow : 98-110.

AZZAROLLI A. (1970) - Villafranchian correlations based on large mammals. Giorn. Geol., Bologna, 35 : 111-131.

BERGGREN W.A. \& VAN COUVERING T.A. (1974) - The Late Neogene. Palaeogeogr., Palaeoclimatol., Palaeoecol., Amsterdam, $16: 1-216$.

BENDA L., MEUlenKamp J.E. \& VAN DE WEERS A. (1977) - Biøstratigraphic correlations in the Eastern Mediterranean Neogene. 3. Correlation between mammals, sporomorph and marine microfossil assemblages from the Upper Cenozoic of Rhodos Greece. Newsl. Stratig., Berlin, 6, 2 : 117-130.

Cabrera Millet M., Lopez Martinez N. \& MiChaUX J. (1983) - Un exemple de lignée endémique ibéroccitane, les campagnols Microtus brecciensis et $M$. cabrerae (Mammalia, Rodentia) : étude phylogénétique et contexte écologique d'un phénomène évolutif récent. BUfFETAUT E., MAZIN J.M. \& SALMON E. (eds). Proc. Int. Symp. George Cuvier, Montbéliard : 69-83.

CHALINE J. (1974) - Un nouveau critère d'étude des Mimomys, et les rapports de Mimomys occitanus - Mimomys stehlini et de Mimomys polonicus (Arvicolidae, Rodentia). Acta Zool. Cracov., 19, 16 : 337-356.

CHALiNE J. (1984) - La séquence des rongeurs de Bresse, en tant que référence biostratigraphique et paléoclimatique. Géologie de la France, Paris, 3 : 251-268.
CHALINe J. \& LAURIN B. (1986) - Phyletic gradualism in a European Plio-Pleistocene Mimonys lineage (Arvicolide, Rodentia). Paleobiology, Chicago, 12, 2 : 203-216.

CHALINE J. \& MiCHAUX J. (1973-74) - Les micromammifères et la biostratigraphie de la bordure occidentale du remplissage bressan (Villafranchien). Bull. Scient. Bourgogne, Dijon, 29 : 55-83.

ESTEBAN J. (1985) - Estudio paleontológico de los Arvicólidos (Rodentia, Mammalia) del Plioceno superior del yacimiento de Casablanca I (Almenara, Castellon). Thèse Lic. Univ. Compl., Madrid, 105 p.

FEJFAR O. \& HEINRICH W.-D. (1981) - Zur biostratigraphischen Untergliederung des Kontinentalen Quartärs in Europa anhand von Arvicoliden (Rodentia, Mammalia). Eclogae Geol. Helv., Basel, 74 : 997-1006.

FEJFAR O. \& HEINRICH W.-D. (1983) - ArvicolidenSukzession und Bioestratigraphie des Oberpliozäns und Quartärs in Europa. In : «Wirbeltier-Evolution und Faunenwandel im Känozoikum». Schriftenr. geol. Wiss., Berlin, 19/20 : 61-109.

FEJFAR o. \& HORÁCEK Il (1983) - Zur Entwicklug der Kleinsäugerfaunen im Villányium und Alt-Biharium auf dem Gebiet der CSSR. Schriftenr. geol. Wiss., Berlin, 19/20 : 111-207.

Freudenthal M., MEIJER T. \& VAN DER MAULEN J. (1976) - Preliminary report on a field campaign in the continental Pleistocene of Tegelen (The Netherlands). Scripta Geologica, Leiden, 34, 27 p.

GIL E. \& SESE C. (1984) - Micromamiferos del nuevo yacimiento Villafranquiense de Casablanca I (Almenara, Prov. de Castellón). Estudios Geol., Madrid, 40: 243-249.

GIL E. \& SESE C. (1985) - Micromamiferos (Insectivora, Rodentia y Lagomorpha) del nuevo yacimiento Villafranquiense de Casablanca B (Almenara, prov. de Castellón). Estudio geol., Madrid, 41 : 945-501.

HELLER F. (1936) - Eine oberpliozäne Wierbel tierfauna aus Rheinhessen. N. Jahrb. fur Miner., Stuttgart, 76 : 99-160.

HINTON M. (1926) - Monograph of the Voles and Lemmings (Microtinae) living and extinct. Brit. Mus. Nat. Hist., London, 448 p.

JANOSSY D. \& VAN DER MEULEN A. (1975) - On Mimomys (Rodentia) from Osztramos 3, North-Hungary. Kon. Nederl. Akad. Wetensch. Proc., B, AmsterdamLondon, 78 : 381-391. 
KOENIGSWALD W.-VON (1980) - Schmelzstriktur und Morphologie in del Molaren der Arvicolidae (Rodentia). Senckenb. Naturfosch. Ges., Frankfurt, $539: 1-129$.

KORMOS Th. (1934) - Neue Insektenfresser, Fledermäuse und Nager aus dem Oberpliozän der Villanyer Gegeng. Földt. Közl., Budapest, 64 : 296-321.

KOVALSKI K. (1958) - An early pleistocene fauna of small mammals form the Kadzielnia Hill in Kielce (Poland). Acta Paleont. Pol., Warszawa, 3, 1 : 1-47.

KRETZOI M. (1953-54) - Bericht über die calabrische (Villafrankische) fauna von Kislang. Jahresber Ung. Geol. Anst., Budapest : 212-265.

KRETZOI M. (1909) - Sketch of the Late Cenozoic (Pliocene and Quaternary) terrestrial stratigraphy of Hungary. Földt. Közl., Budapest, 3 : 179-204.

MEIN P. (1975) - Biozonation du Néogène Méditerranéne à partir des Mammifères. IUGS, RCMNS. Report on Activity of the RCMNS Working Groups, Bratislava : 78-81.

MEIN P., MOISSENET E. \& TRUC G. (1978) - Les formations continentales du Néogène supérieur des vallées du Júcar et du Cabriel au NE d'Albacete (Espagne). Biostratigraphie et environnement. Doc. Lab. Géol. Univ. Lyon, 72 : 99-147.

MichaUX J. (1970) - Les Rongeurs (Arvicolidés, Muridés et Gliridés) de la localité Arondelli à Villafranca d'Asti (Italie), Palaeont. Italica, Pisa, 66, $36:$ 67-80.

MICHAUX J. (1971) - Arvicolinae (Rodentia) du Pliocène terminal et du Quaternaire ancien de France et d'Espagne. Palaeovertebrata, Montpellier, 4, 4 : 137-214.

PARETTO M.F. (1865) - Note sur la subdivision que l'on pourrait établir dans les terrains tertiaires de l'Appenin septentrional. Bull. Soc. géol. France, Paris, (2), 22 : 210-277.

RABEDER G. (1981) - Die Arvicoliden (Rodentia, Mammalia) aus dem älteren Pleistozän von Niederösterreich. Beitr. Paläont. Osterr., Wien, $8: 343$ p.

RABEDER G. \& SATTLER F. (1984) - Eine neue und rationelle Methode zur Untersuchung von Schmelzstrukturen bei Wühlmaus-Zähnen. Der Präparator, Bochum, 30, $2: 275-281$

TAUXe L., Opdyke N.D., PASINI G. \& Elmi C. (1981) - Age of the Plio-Pleistocene boundary in the Vrica section, southern Italy. Nature, London, $304:$ 125-129.

THALER L. (1966) - Les Rongeurs fossiles du Bas-Languedoc dans leurs rapports avec l'histoire des faunes et la stratigraphie du Tertiaire d'Europe. Mém. Mus. Nat. Hist. Nat., N.S., Sér. C, Paris, 17, 295 p.

VAN DER MEULEN A.J. (1973) - Middle Pleistocene smaller Mammals from the Monte Peglia [Orvieto, Italy (with special reference to the phylogeny of Mcrotys (Arvicolidae, Rodentia)]. Quaternaria, Roma, 17, 144 p.

VAN DER MEUlen A.J. (1974) - On Microtus (Allophaiomys) deucalion (KRETZOI, 1969), (Arvicolidae, Rodentia), from the Upper Villányan (Lower Pleistocene) of Villány-5. S. Hungary. Koninkl. Nederl. Akad. Wetens., Amsterdam, Ser. B, $3: 259-266$.

VAN DER MEULEN A.J. (1978) - Microtus and Pitymys (Arvicolidae from Cumberland Cave, Maryland. With a comparison of some new and old world species. Ann. Carnegie Mus., Pittsburg, 47, 6 : 101-145.

VAN DE WEERD A. (1976) - Rodent Faunas of the MioPliocene Continental Sediments of the Teruel-Alfambra Region, Spain. Utrecht Micropal. Bull., Spec. Publ.; Utrecht, $2: 217 \mathrm{p}$. 


\section{PLANCHE 1}

\section{Mimomys tornensis JANOSSY \& V. MEULEN, 1975 de CSB I, niveau A}

Fig. 1 à $5-\mathrm{M}_{1}:$ RA1, RA2, RA5, RA7, RA10.

Fig. $6,7-\mathrm{M}_{2}$ : RA26, RA28.

Fig. 10, 11, $12-\mathrm{M}^{1}$ : RA54, RA57, RA60.

Fig. 13, $14-\mathrm{M}^{2}$ : RA67, RA72.

Fig. $15,16,17,18-M^{3}$ : RA74, RA75, RA78, RA79. 


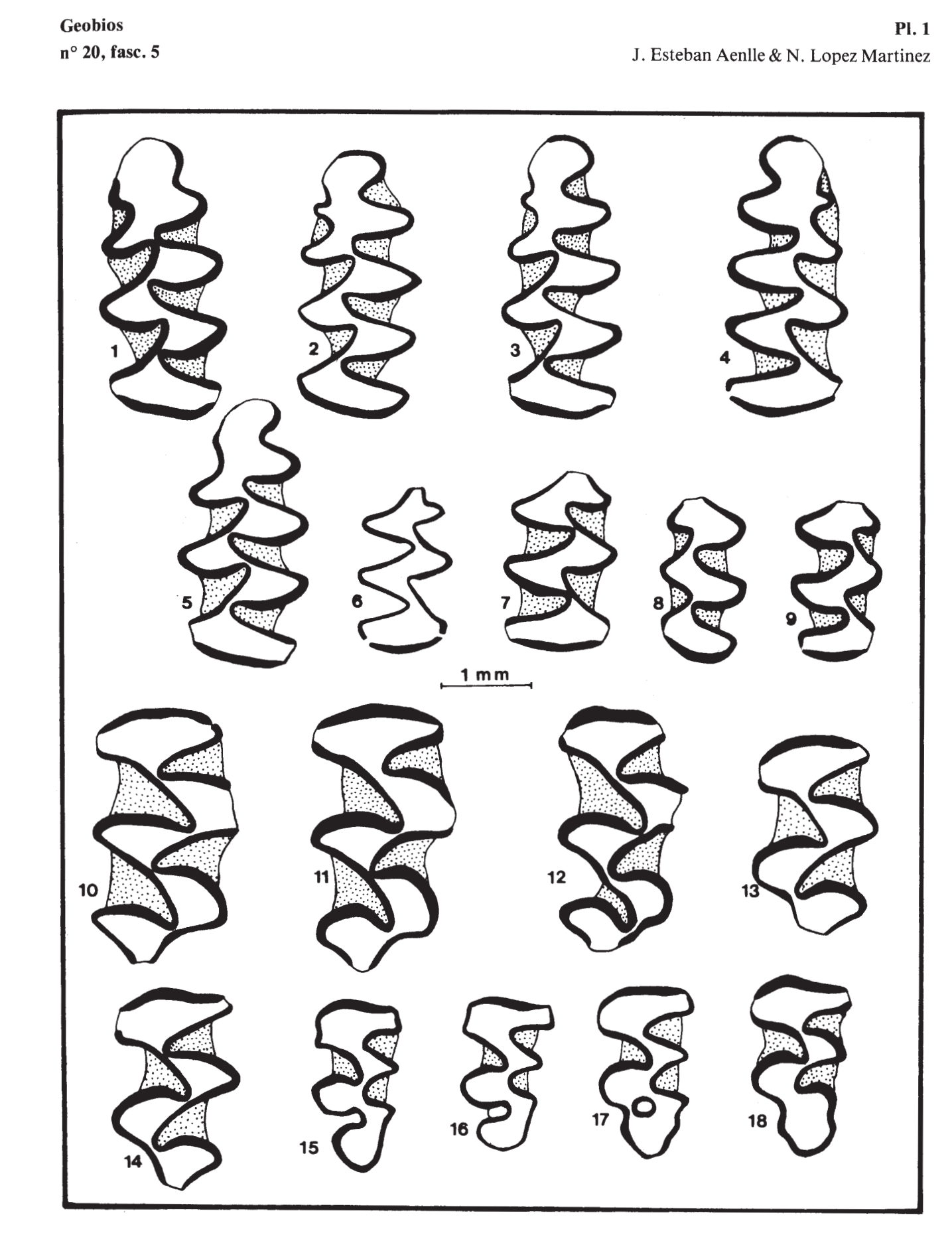




\section{PLANCHE 2}

Mimomys medasensis MICHAUX, 1971 de CSB I, niveau A.

Fig. 1 à $7-M_{1}$ : RA90, RA82, RA87, RA85, RA91, RA93, RA94.

Fig. 8, 9, $10-\mathrm{M}_{2}$ : RA275, RA279, RA108.

Fig. 11, 12, $13-\mathrm{M}_{3}$ : RA310, RA315, RA130.

Fig. 14, 15, $16-\mathrm{M}^{1}$ : RA339, RA160, RA342.

Fig. 17, 18, $19-\mathrm{M}^{2}$, RA180, RA381, RA383.

Fig. $20,21-\mathrm{M}^{3}$ : RA402, RA407. 

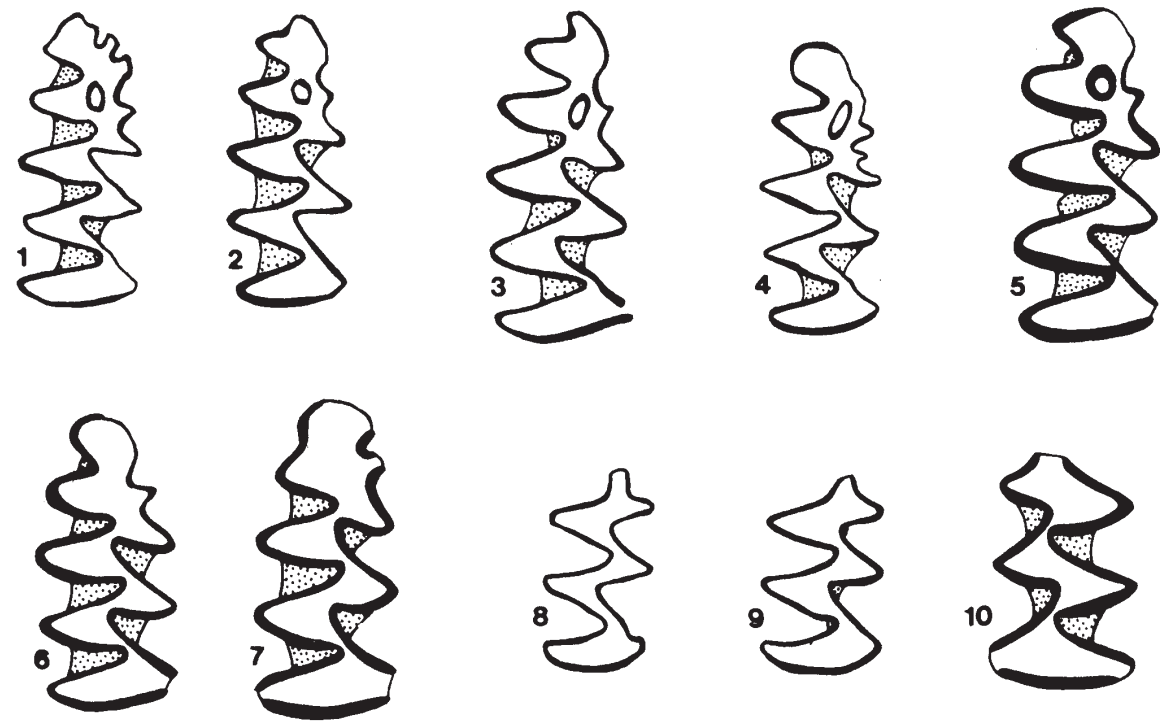

$1 \mathrm{~mm}$
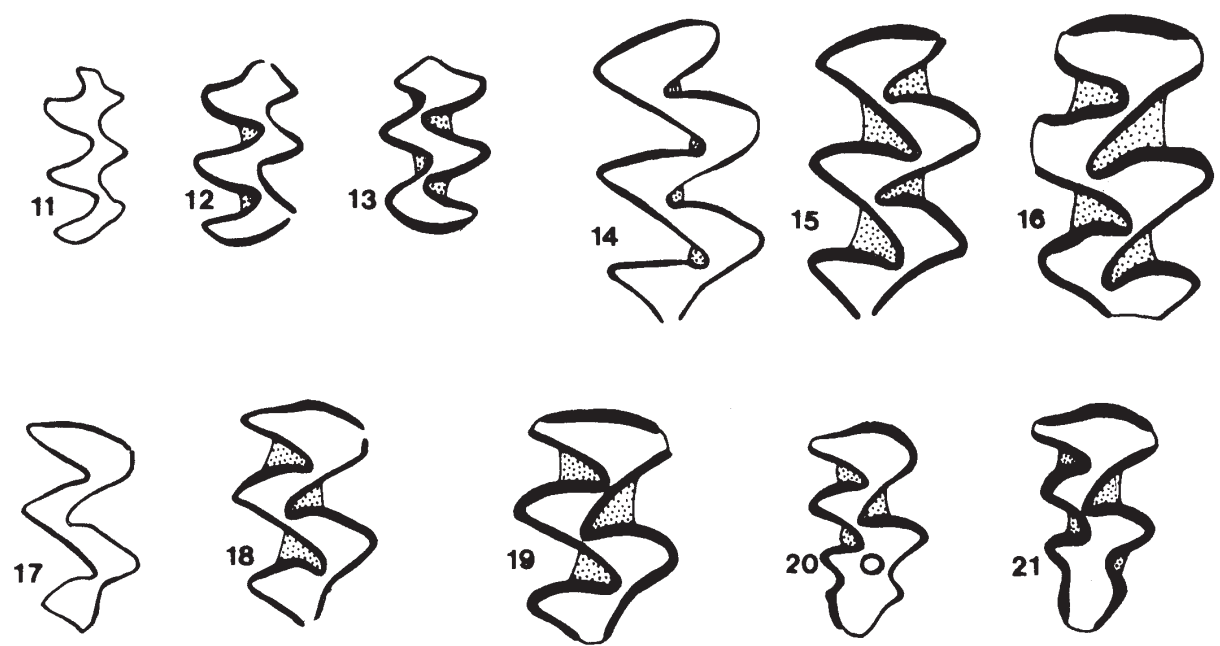


\section{PLANCHE 3}

\section{Mimomys aff. rex KORMOS, 1934 de CSB I}

Fig. 1 à $5-M_{1}:$ RA219, RA420, RA218, RA422, RA217.

Fig. $6,7,8-\mathrm{M}_{2}$ : RA439, RA441, RA447.

Fig. 9, $10-\mathrm{M}^{2}$ : RA456, RA459.

Fig. $11,12,13-M_{2}:$ RA468, RA469, RA471.

Fig. $14,15,16-\mathrm{M}^{2}$ : RA475, RA477, RA481.

Fig. $17,18,19-\mathrm{M}^{3}$ : RA485, RA487, RA489. 

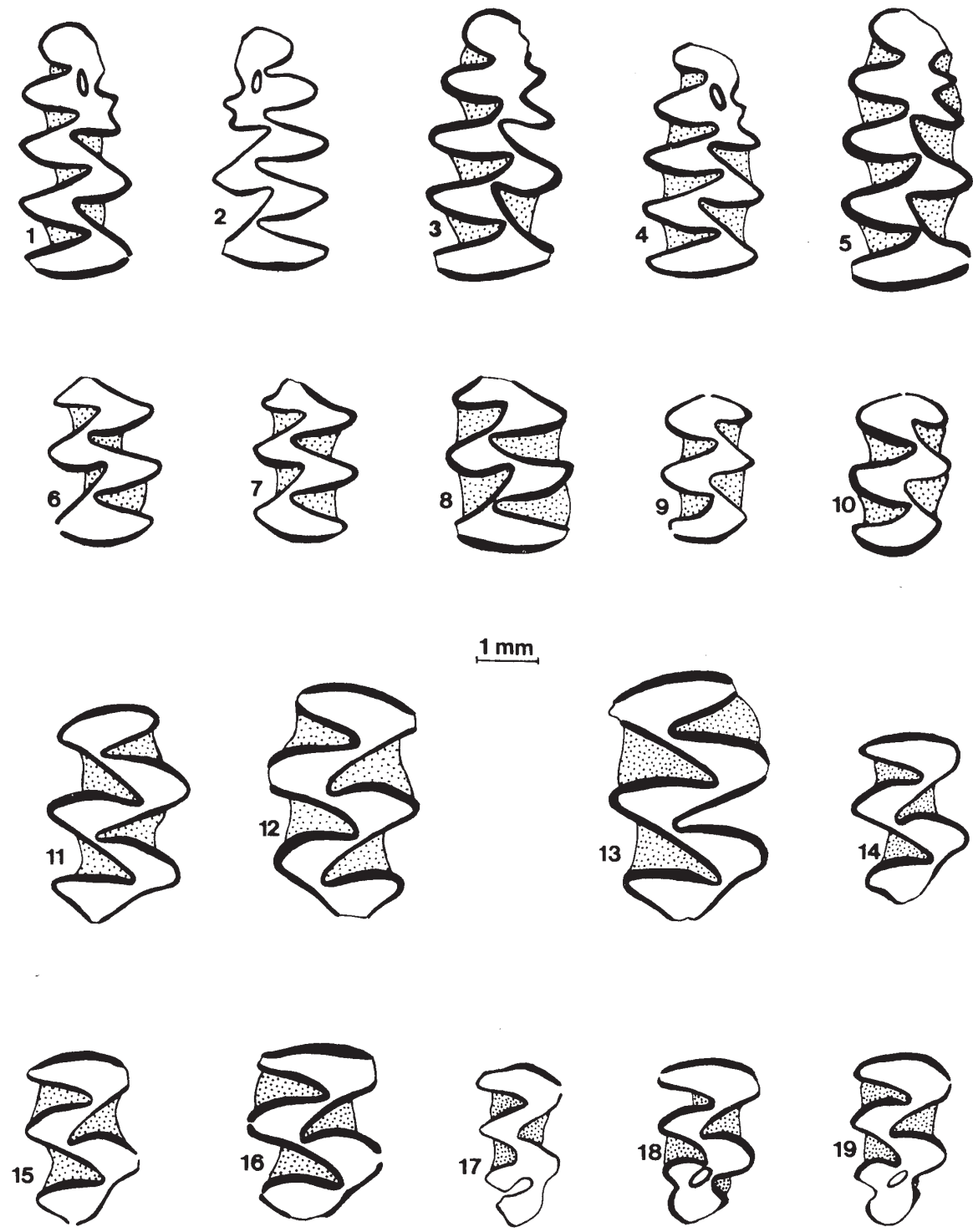


\title{
PLANCHE 4
}

\section{Structure de l'émail chez la $M_{1}$ de $M$. aff. rex de CSB I.}

Enamel pattern of the Mimomys aff. rex $\mathrm{M}_{1}$ from CSB I.

\begin{abstract}
a) Bord avant, x 375 environ. Dans la partie supérieure, émail radial : en dessous, émail lamellaire.
a) Leading edge, ca. x 375 . Above, radial enamel ; below, lamellar enamel.
\end{abstract}

b) Vue du T3 x 170 environ. On observe l'émail radial changer de la partie externe dans le bord d'avant à la partie interne dans le bord d'arrière.

b) Pattern of the T3, ca. $\times 170$. Radial enamel changes from the outer part of the leading edge, to the inner part of the trailing edge.

c) Bord arrière, x 375 environ. Dans la partie supérieure, émail radial, en dessous émail tangentiel.

c) Trailing edge, ca. x 375. Above, radial enamel ; below, tangential enamel. 


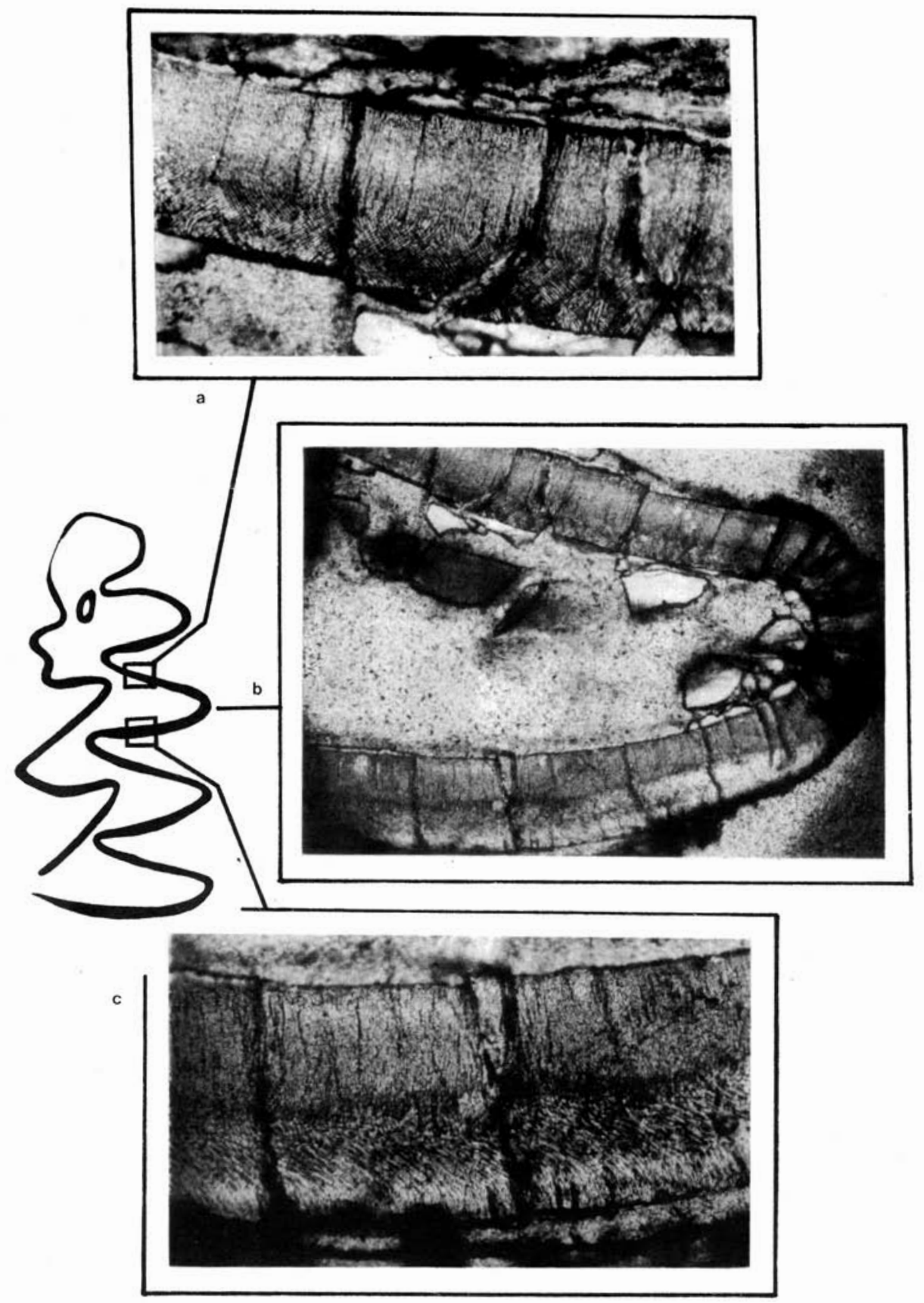

